\title{
Mechanisms underlying adverse effects of HDL on eNOS-activating pathways in patients with coronary artery disease
}

Christian Besler, 1,2,3 Kathrin Heinrich,1,2,3 Lucia Rohrer, ${ }^{3,4}$ Carola Doerries,, 1,2,3 Meliana Riwanto,1,2,3 Diana M. Shih, ${ }^{5}$ Angeliki Chroni, ${ }^{6}$ Keiko Yonekawa, ${ }^{1,2}$ Sokrates Stein, ${ }^{1,2,3}$ Nicola Schaefer, ${ }^{1,2,3}$ Maja Mueller,,1,2 Alexander Akhmedov, ${ }^{1,2,3}$ Georgios Daniil, ${ }^{1}$ Costantina Manes,,1,2 Christian Templin,1,2 Christophe Wyss,1,2 Willibald Maier,1,2 Felix C. Tanner,1,2,3 Christian M. Matter, ${ }^{1,2,3}$ Roberto Corti, ${ }^{1,2}$ Clement Furlong, ${ }^{7}$ Aldons J. Lusis, ${ }^{5}$ Arnold von Eckardstein,3,4 Alan M. Fogelman,5 Thomas F. Lüscher, 1,2,3 and Ulf Landmesser1,2,3

\begin{abstract}
${ }^{1}$ Cardiology, Cardiovascular Center, University Hospital Zurich, Zurich, Switzerland. ${ }^{2}$ Cardiovascular Research, Institute of Physiology, and ${ }^{3}$ Center for Integrative Human Physiology, University of Zurich, Zurich, Switzerland. ${ }^{4}$ Institute of Clinical Chemistry, University Hospital Zurich, Zurich, Switzerland. ${ }^{5}$ David Geffen School of Medicine, UCLA, Los Angeles, California, USA. ${ }^{6}$ nnstitute of Biology, National Center Scientific Research "Demokritos," Athens, Greece. ${ }^{7}$ Departments of Medicine (Division of Medical Genetics) and Genome Sciences, University of Washington, Seattle, Washington, USA.
\end{abstract}

\begin{abstract}
Therapies that raise levels of HDL, which is thought to exert atheroprotective effects via effects on endothelium, are being examined for the treatment or prevention of coronary artery disease (CAD). However, the endothelial effects of HDL are highly heterogeneous, and the impact of HDL of patients with CAD on the activation of endothelial eNOS and eNOS-dependent pathways is unknown. Here we have demonstrated that, in contrast to HDL from healthy subjects, HDL from patients with stable CAD or an acute coronary syndrome $\left(\mathrm{HDL}_{\mathrm{CAD}}\right)$ does not have endothelial antiinflammatory effects and does not stimulate endothelial repair because it fails to induce endothelial NO production. Mechanistically, this was because $H_{D L} L_{C A D}$ activated endothelial lectin-like oxidized LDL receptor 1 (LOX-1), triggering endothelial PKC $\beta I I$ activation, which in turn inhibited eNOS-activating pathways and eNOS-dependent NO production. We then identified reduced HDL-associated paraoxonase 1 (PON1) activity as one molecular mechanism leading to the generation of HDL with endothelial PKC $\beta I I$-activating properties, at least in part due to increased formation of malondialdehyde in HDL. Taken together, our data indicate that in patients with CAD, HDL gains endothelial LOX-1- and thereby PKC $\beta$ II-activating properties due to reduced HDL-associated PON1 activity, and that this leads to inhibition of eNOS-activation and the subsequent loss of the endothelial antiinflammatory and endothelial repair-stimulating effects of $\mathrm{HDL}$.
\end{abstract}

\section{Introduction}

Low levels of HDL cholesterol are associated with an increased risk of coronary artery disease (CAD) (1). Furthermore, in patients with $\mathrm{CAD}$ and low levels of LDL cholesterol receiving statin treatment, low HDL cholesterol levels were predictive of an elevated risk of major cardiovascular events (2).

Notably, besides promotion of cholesterol efflux from macrophage foam cells $(3,4)$, HDL has been found to exert several important endothelial antiatherogenic effects $(5,6)$. In particular, several recent studies have indicated that HDL stimulates endothelial cell NO production (7-10) and promotes endothelial repair mechanisms (11-15). These protective effects of HDL on eNOS and endothelial repair are, at least in part, mediated via endothelial scavenger receptor B, type I (SR-BI) $(7,8,11)$ and involve cholesterol efflux and the adaptor molecule PDZK1 $(16,17)$. Moreover, by binding to SR-BI, HDL has been reported to activate eNOS via stimulation of the phosphorylation of eNOS at Ser1177, induced via PI3K and Akt kinase (8). In addition, HDL has been reported to partly stimulate eNOS-activating phosphorylation at Ser1177 and endothelial NO production via endothelial sphingosine-1-

Conflict of interest: Alan M. Fogelman is a principal and officer in Bruin Pharma. Citation for this article: J Clin Invest. 2011;121(7):2693-2708. doi:10.1172/JCI42946. phosphate receptor $3\left(\mathrm{~S}_{1} \mathrm{P}_{3}\right)(9)$. Moreover, recent studies in mice have demonstrated that HDL protects against dietary cholesterol-induced endothelial dysfunction by promoting cholesterol efflux and release of 7-ketocholesterol via the endothelial ABCG1 transporter, and reduced hypercholesterolemia-induced interaction of caveolin-1 with eNOS $(10,18)$. In humans with hypercholesterolemia or with isolated low HDL levels due to heterozygous $A B C A 1$ gene mutations, administration of reconstituted HDL has been observed to normalize impaired endothelium-dependent, NO-mediated vasodilation $(19,20)$. These endothelial-protective effects of HDL are thought to contribute importantly to its antiatherogenic functions (21). Indeed, endothelial dysfunction, in particular reduced endothelial NO bioavailability, is considered to play a key role in the initiation and progression of atherosclerosis and its clinical complications (22-27).

Based on epidemiological studies and observations of potential atheroprotective properties of HDL, HDL-raising interventions are currently being intensely evaluated as a novel therapeutic strategy in patients with CAD (28-30). The adverse effects of the cholesteryl ester transfer protein (CETP) inhibitor torcetrapib on cardiovascular outcome (31), which were observed despite a substantial increase in HDL levels, have suggested that a better understanding of the vascular effects of HDL from patients with $\mathrm{CAD}$ is urgently required. 
Table 1

Characteristics of the study population

\begin{tabular}{|c|c|c|c|c|}
\hline & $\begin{array}{l}\text { Healthy subjects } \\
\qquad(n=25)\end{array}$ & $\begin{array}{c}\text { SCAD } \\
(n=25)\end{array}$ & $\begin{array}{c}\text { ACS } \\
(n=25)\end{array}$ & $P$ \\
\hline Age (yr) & $54 \pm 5$ & $56 \pm 6$ & $54 \pm 6$ & NS \\
\hline Sex (male/female) & $19 / 6$ & $19 / 6$ & $18 / 7$ & NS \\
\hline BMI $\left(\mathrm{kg} / \mathrm{m}^{2}\right)$ & $26 \pm 5$ & $27 \pm 4$ & $26 \pm 3$ & NS \\
\hline $\mathrm{MAP}(\mathrm{mmHg})$ & $96.6 \pm 6.4$ & $96.4 \pm 13.8$ & $96.1 \pm 12.8$ & NS \\
\hline Fasting glucose (mmol/l) & $5.3 \pm 1.0$ & $5.4 \pm 0.9$ & $5.1 \pm 0.7$ & NS \\
\hline $\mathrm{HbA} 1 \mathrm{c}(\%)$ & $5.5 \pm 0.2$ & $5.4 \pm 0.6$ & $5.7 \pm 0.9$ & NS \\
\hline Total cholesterol (mmol/l) & $4.8 \pm 0.7$ & $4.3 \pm 1.2$ & $4.5 \pm 0.6$ & NS \\
\hline HDL cholesterol (mmol/l) & $1.4 \pm 0.4$ & $1.2 \pm 0.3$ & $1.2 \pm 0.9$ & NS \\
\hline LDL cholesterol (mmol/l) & $2.9 \pm 0.9$ & $2.7 \pm 1.0$ & $2.8 \pm 0.6$ & NS \\
\hline Triglycerides (mmol/l) & $1.1 \pm 0.4$ & $1.2 \pm 0.3$ & $1.1 \pm 0.2$ & NS \\
\hline $\operatorname{CRP}(\mu \mathrm{mol} / \mathrm{l})$ & $2.0 \pm 1.0$ & $3.0 \pm 5.0$ & $4.0 \pm 8.0$ & NS \\
\hline Creatinine $(\mu \mathrm{mol} / \mathrm{l})$ & $88.0 \pm 9.0$ & $89.0 \pm 21.0$ & $84.0 \pm 10.0$ & NS \\
\hline ASS (\%) & 0 & 100 & 80 & 0.03 \\
\hline Clopidogrel (\%) & 0 & 40 & 32 & NS \\
\hline ACE-I/ARB (\%) & 0 & 68 & 56 & NS \\
\hline Beta blocker (\%) & 0 & 68 & 52 & 0.04 \\
\hline Diuretics (\%) & 0 & 12 & 16 & NS \\
\hline Calcium blocker (\%) & 0 & 8 & 8 & NS \\
\hline Statins (\%) & 0 & 76 & 64 & NS \\
\hline
\end{tabular}

CRP, C-reactive protein; ACE-I, angiotensin-converting enzyme inhibitor; ARB, angiotensin II type I receptor blocker; ASS, acetylsalicylic acid (aspirin).

characteristics of the study population are shown in Table 1.

Effects of $H D L_{C A D}$ as compared with $H D L_{\text {Healthy }}$ on endothelial NO production. Stimulation of endothelial NO production has been suggested as an important endothelial-atheroprotective effect of HDL $(7-10,18,21,36)$. HDL $_{\text {Healthy }}$ stimulated endothelial cell NO production as observed by both electron spin resonance (ESR) spectroscopy analysis and measurements using the fluorescent probe 4,5-diaminofluorescein (Figure 1, A and B, and Supplemental Figure 1, A and B; supplemental material available online with this article; doi:10.1172/JCI42946DS1). In contrast to HDL from healthy subjects, HDL from patients with sCAD failed to stimulate endothelial NO production, and HDL from patients with ACS very modestly inhibited endothelial NO production (Figure 1, A and B, and Supplemental Figure 1, $A$ and $B)$. Increasing concentrations of HDL $_{\text {Healthy }}$ further augmented endothelial cell NO production, whereas no such effect was observed in response to increasing concentrations of $\mathrm{HDL}_{\mathrm{CAD}}$ (Figure 1C). The effects of $\mathrm{HDL}_{\mathrm{Healthy}}$ on endothelial cell $\mathrm{NO}$

Indeed, the capacity of HDL to mediate cholesterol efflux from lipid-laden macrophages has been observed to be impaired after substantial oxidative modification of HDL by myeloperoxidase or malondialdehyde, a lipid peroxidation product, as observed in atherosclerotic plaques $(32,33)$. In addition, the capacity of HDL to prevent the proinflammatory effects of LDL, i.e., LDL-induced monocyte chemotactic activity (34), was impaired in patients with CAD (35). Furthermore, the effects of HDL on endothelial NO bioavailability can be highly variable, as observed in patients with type 2 diabetes or antiphospholipid syndrome (APS) $(36,37)$; however, the underlying mechanisms remain to be determined. Interestingly, we have observed that reduced serum activity of paraoxonase 1 (PON1), an HDL-associated enzyme, was related to endothelial dysfunction in patients with APS (37); however, whether PON1 plays a causal role in the effects of HDL on endothelial NO production remains unknown. Moreover, as described above, patients with CAD represent a major target population for HDL-raising therapies, and whether the effects of HDL from these patients on endothelial NO production and eNOS-dependent atheroprotective pathways are altered remains to be determined.

The present study was therefore designed to compare the effects of HDL from patients with stable CAD (sCAD) or acute coronary syndrome (ACS) with those of HDL from healthy subjects on eNOS-activating or -inhibiting pathways and endothelial NO production, and to investigate the role of HDL-induced NO production for the capacity of HDL to inhibit endothelial antiinflammatory activation and to stimulate endothelial repair. In particular, we focused on the characterization of the mechanisms underlying altered effects of HDL from patients with CAD on endothelial cell NO production and eNOS-dependent pathways.

\section{Results}

HDL was isolated from patients with sCAD or ACS (HDL $\mathrm{HAD}_{\mathrm{CAD}}$ and age- and sex-matched healthy subjects ( $\left.\mathrm{HDL}_{\mathrm{Healthy}}\right)$. The production were modest, but in a similar range as compared with the effects of bradykinin (Supplemental Figure 1C), a known stimulus of endothelial cell NO production (38). In addition, both HDL subfractions $\left(\mathrm{HDL}_{2}\right.$ and $\left.\mathrm{HDL}_{3}\right)$ from patients with sCAD or ACS (Figure 1D) had an impaired capacity to stimulate endothelial NO production.

Effects of $H D L_{\text {Healthy }}$ and $H D L_{C A D}$ on eNOS-activating pathways and endothelial superoxide production. We next compared the effects of $\mathrm{HDL}_{\mathrm{Healthy}}$ and $\mathrm{HDL}_{\mathrm{CAD}}$ on eNOS-activating pathways and endothelial superoxide production, which may limit NO bioavailability $(23,39)$. Activation of eNOS in response to $\mathrm{HDL}_{\mathrm{Healthy}}$ has been shown to be dependent on Akt-mediated eNOS Ser1177 phosphorylation $(8,9)$, a well-known eNOS-activating pathway (40). We observed that $\mathrm{HDL}_{\text {Healthy }}$ but not $\mathrm{HDL}_{\mathrm{CAD}}$ stimulated endothelial Akt phosphorylation at Ser473 and Akt-dependent phosphorylation of eNOS at Ser1177 (Figure 2, A and B). Moreover, the inhibitory phosphorylation of eNOS at Thr495 (38) was increased by HDL $\mathrm{CAD}_{\mathrm{C}}$ (Figure $2 \mathrm{C}$ ). Time-course experiments demonstrated a statistically significant difference in endothelial Akt phosphorylation at Ser473 and eNOS phosphorylation at Ser1177 and Thr495 at different time points after exposure of endothelial cells to either $\mathrm{HDL}_{\mathrm{Healthy}}$ or $\mathrm{HDL}_{\mathrm{CAD}}$ (Supplemental Figure 2, A-C). Whereas stimulation of eNOS Ser1177 phosphorylation is involved in the activation of the enzyme $(8,9)$, there is no clear evidence that a reduction in eNOS Ser1177 phosphorylation, as observed in response to HDL from patients with ACS (Figure 2B), is functionally relevant.

In addition, we characterized the effects of HDL on endothelial

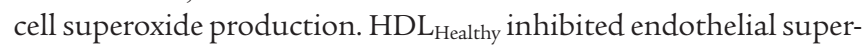
oxide production (Figure 2D) and reduced endothelial NAD $(\mathrm{P}) \mathrm{H}$ oxidase activity (Figure 2, E and F), whereas $\mathrm{HDL}_{\mathrm{CAD}}$ did not significantly reduce endothelial superoxide production or $\mathrm{NAD}(\mathrm{P}) \mathrm{H}$ oxidase activity (Figure 2, D-F). Importantly, we did not observe 

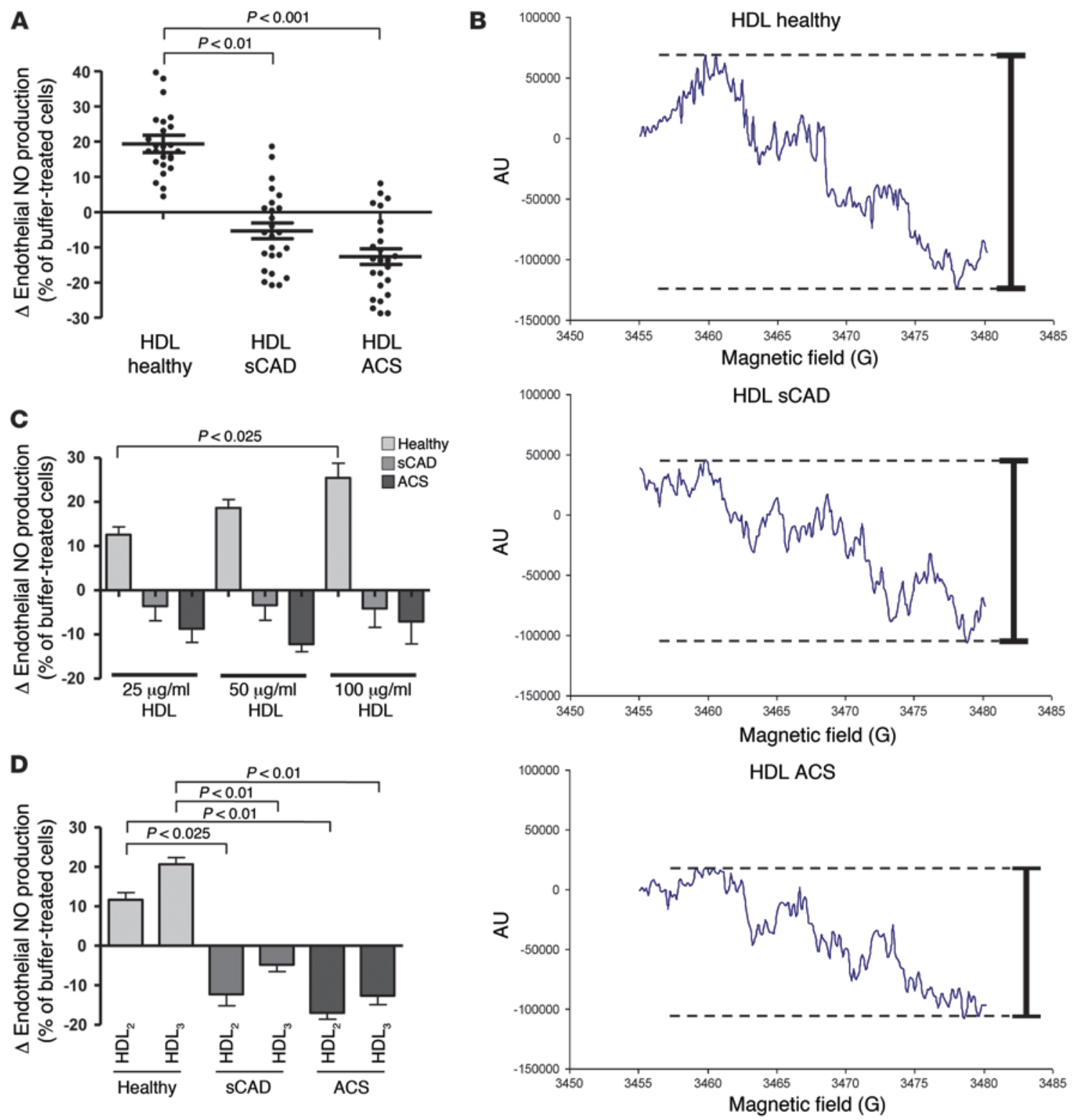

Figure 1

Effects of HDL from healthy subjects, patients with SCAD or ACS on endothelial cell NO production, as determined by ESR spectroscopy analysis. (A) HDL from healthy subjects and patients with SCAD or ACS was isolated by sequential ultracentrifugation, and the effects of HDL (50 $\mu \mathrm{g} / \mathrm{ml}$ ) on endothelial cell NO production were analyzed by ESR spectroscopy analysis ( $n=25$ per group). Data are expressed as percent change versus buffer-treated cells; data points for each study participant are shown. (B) Representative ESR spectra of the NO-Fe(DETC) ${ }_{2}$ signal in endothelial cells treated with HDL from healthy subjects and patients with SCAD or ACS. (C) Increasing physiological concentrations of HDL (25, 50 , and $100 \mu \mathrm{g} / \mathrm{ml}$ ) from healthy subjects stimulated endothelial NO production that was not observed with increasing concentrations of HDL from patients with SCAD or ACS ( $n=12$ per group). (D) Effects of $\mathrm{HDL}_{2}(d=1.063-1.125 \mathrm{~g} / \mathrm{ml})$ and HDL $3(d=1.125-1.21 \mathrm{~g} / \mathrm{ml})$ from patients with SCAD or ACS and healthy subjects, separated by sequential ultracentrifugation, on endothelial cell NO production ( $n=12-15$ per group).

an increase in endothelial superoxide production in response to $\mathrm{HDL}_{\mathrm{CAD}}$ as detected by ESR spectroscopy analysis (Figure 2D). Taken together, these data suggest that impaired activation of eNOS-activating pathways rather than stimulation of NO inactivation by superoxide underlies the impaired capacity of $\mathrm{HDL}_{\mathrm{CAD}}$ to increase endothelial NO availability.

Role of eNOS in endothelial antiinflammatory effects of $H D L$. We next examined whether the altered effects of $\mathrm{HDL}_{\mathrm{CAD}}$ on endothelial NO availability affect the ability of HDL to inhibit endothelial
VCAM-1 expression and monocyte adhesion. As shown in Figure 3, A-C, HDL $\mathrm{H}_{\text {Healthy }}$ inhibited nuclear translocation and DNA binding of the p65 subunit of NF- $\mathrm{kB}$, a proinflammatory transcription factor involved in the upregulation of VCAM-1 (41). To determine whether stimulation of eNOS-mediated NO production is important for the endothelial antiinflammatory effects of HDL, we inhibited eNOS protein expression in endothelial cells by using eNOS-specific siRNA silencing (Supplemental Figure 3). Inhibition of eNOS-mediated NO production by both eNOS-spe- 
A

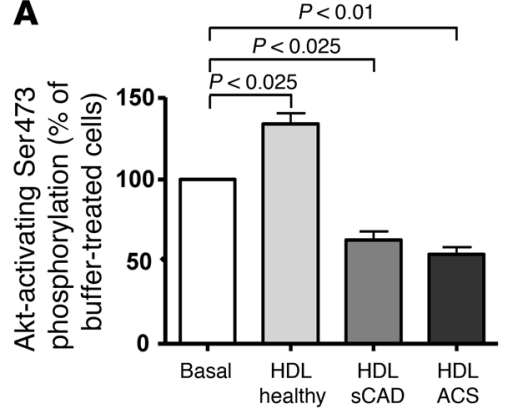

pAkt (Ser473)

Total Akt
B

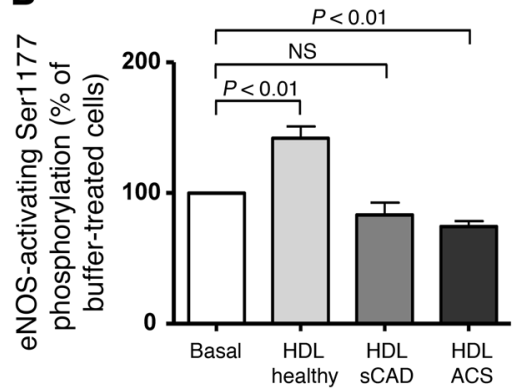

peNOS (Ser1177)

Total eNOS
C

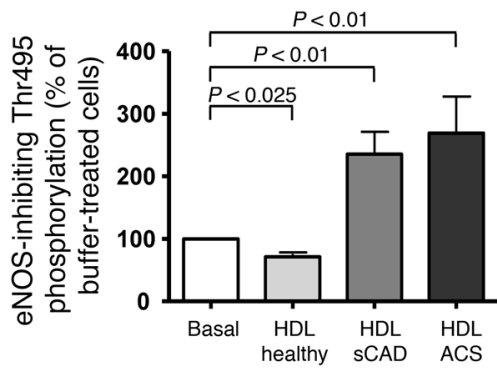

peNOS (Thr495)

Total eNOS
D

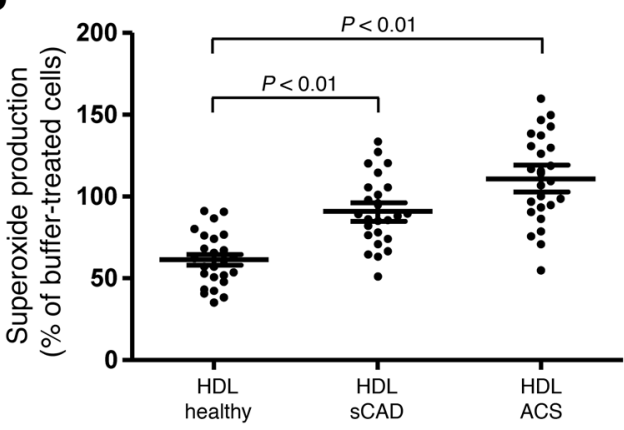

$\mathbf{F}$
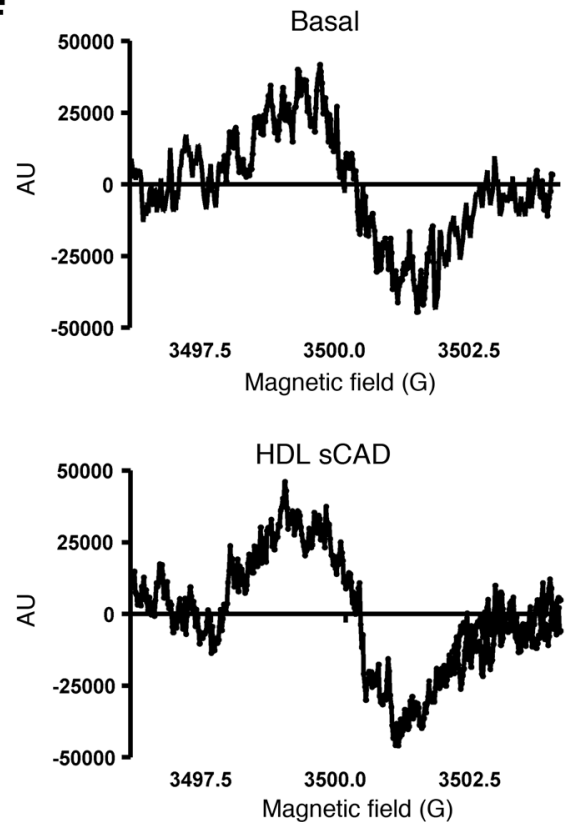

E

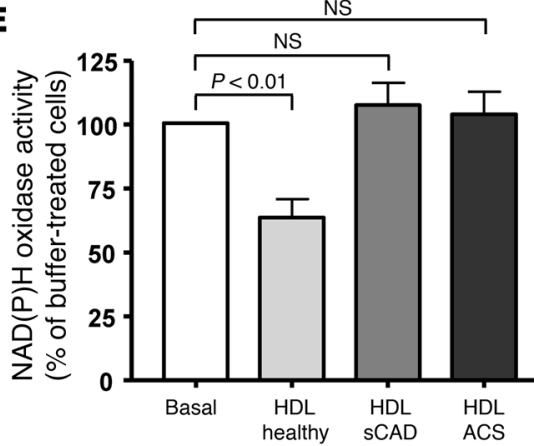

HDL healthy
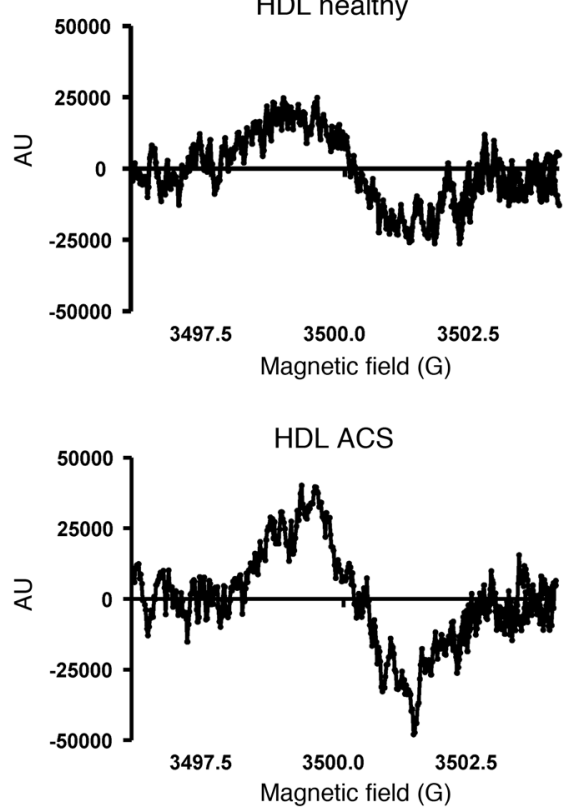

Figure 2

Effects of HDL from healthy subjects and patients with SCAD and ACS on endothelial Akt activation, eNOS-activating/inhibiting phosphorylation, and endothelial superoxide production. (A) Effects of HDL (50 $\mathrm{\mu g} / \mathrm{ml})$ on phosphorylation of Akt at Ser473, (B) Akt-dependent activating phosphorylation of eNOS at Ser1177, and (C) inhibitory phosphorylation of eNOS at Thr495 in HAECs, as detected by Western blot analysis ( $n=10-12$ per group). (D) Effects of HDL on endothelial superoxide production ( $n=25$ per group; data points for all study participants are shown) and $(E)$ NAD $(P) H$ oxidase activity, as measured by ESR spectroscopy ( $n=8-10$ per group). (F) Representative ESR spectra of NAD(P)H oxidase activity in HAECs treated with buffer (basal) and with HDL from healthy subjects or patients with sCAD or ACS. 
A

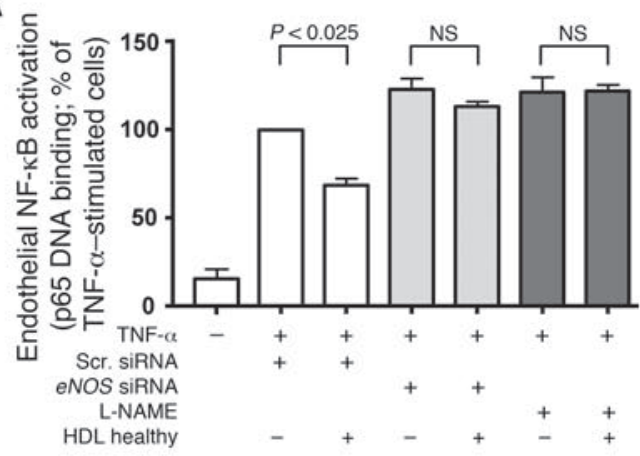

C

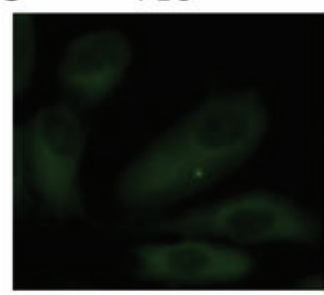

TNF- $\alpha$

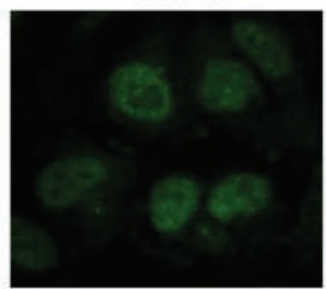

B 든

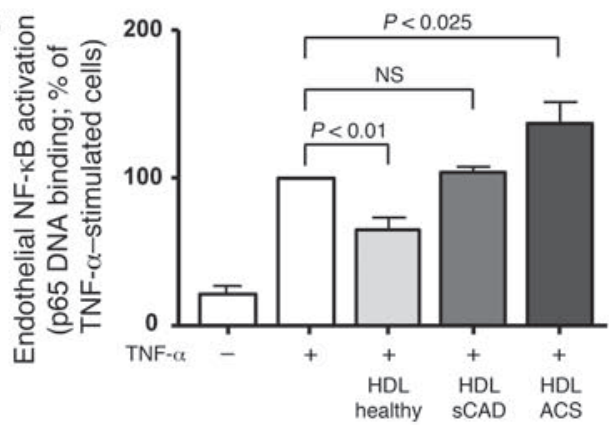

D

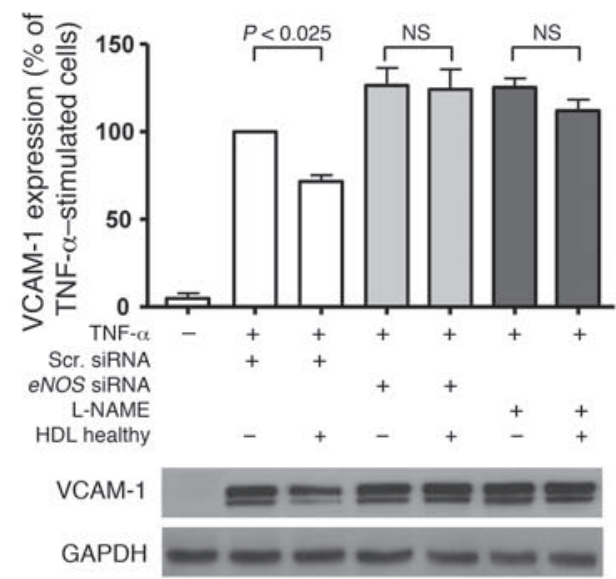

TNF- $\alpha+$ HDL healthy

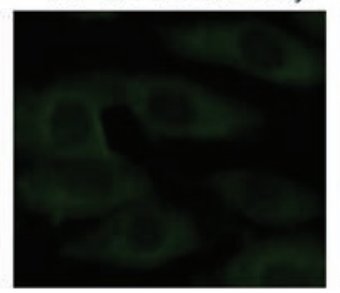

TNF- $\alpha+$ HDL sCAD

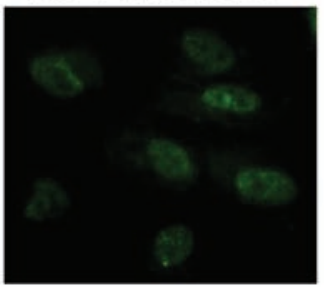

TNF- $\alpha+$ HDL ACS

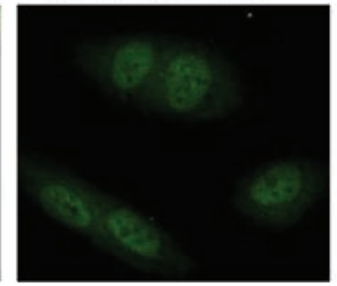

E

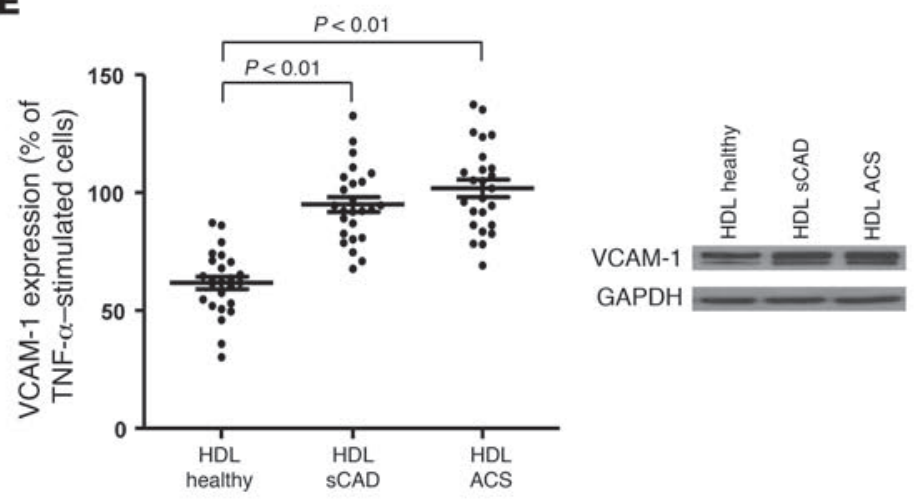

Figure 3

Critical role of eNOS in the effects of HDL on TNF- $\alpha$-stimulated endothelial NF-kB activation and VCAM-1 expression. (A) Inhibition of eNOSmediated endothelial NO production by siRNA knockdown of eNOS or treatment with L-NAME (1 mM) prevented the effects of HDL ( $50 \mu \mathrm{g} / \mathrm{ml})$

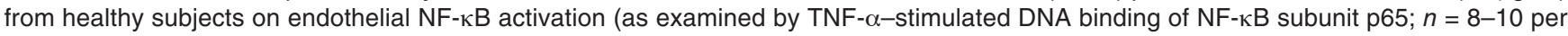
group). Scr., scrambled. (B) In contrast to HDL from healthy subjects, HDL from patients with SCAD and ACS failed to inhibit TNF- $\alpha-$ stimulated endothelial NF-KB activation ( $n=10-12$ per group). (C) Representative images (original magnification, $\times 20)$ showing that HDL from a healthy subject, but not from patients with SCAD or ACS, inhibited endothelial nuclear translocation of NF-kB subunit p65, as detected by fluorescence microscopy of TNF- $\alpha$-stimulated HAECs. (D) Inhibition of eNOS-mediated NO production by siRNA knockdown of eNOS or treatment with L-NAME prevented the effects of HDL from healthy subjects on TNF- $\alpha$-stimulated VCAM-1 expression in HAECs, as detected by Western blot analysis ( $n=8-10$ per group). (E) Effect of HDL from healthy subjects and patients with SCAD or ACS on TNF- $\alpha-$ stimulated VCAM-1 expression in HAECs ( $n=25$ per group; data points for all study participants are shown).

cific RNA interference and pharmacological inhibition by $N$-nitroL-arginine methyl ester (L-NAME, $1 \mathrm{mM}$ ) prevented the inhibitory

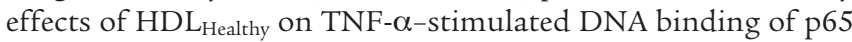
(Figure $3 \mathrm{~A}$ ), suggesting that endothelial NO production plays a crucial role in the inhibition of endothelial NF- $\kappa \mathrm{B}$ activation by HDL. In line with these findings, $\mathrm{HDL}_{\mathrm{CAD}}$ did not prevent TNF- $\alpha-$ stimulated nuclear translocation and DNA binding of the p65 subunit of NF-KB (Figure 3, B and C). In contrast to $\mathrm{HDL}_{\mathrm{Healthy}}, \mathrm{HDL}_{\mathrm{CAD}}$ increased DNA binding of the $\mathrm{p} 65$ subunit of NF-אB in unstimulated endothelial cells (Supplemental Figure 4A). Furthermore, both siRNA interference and pharmacological inhibition of eNOS prevented the inhibitory effects of HDL $\mathrm{Healthy}_{\text {on endothelial VCAM-1 }}$ expression (Figure 3D), suggesting that the stimulatory effect of HDL on endothelial NO production is critical for this endothelial antiinflammatory effect of HDL. Consistent with these findings, HDL from patients with sCAD or ACS failed to inhibit TNF- $\alpha-$ 

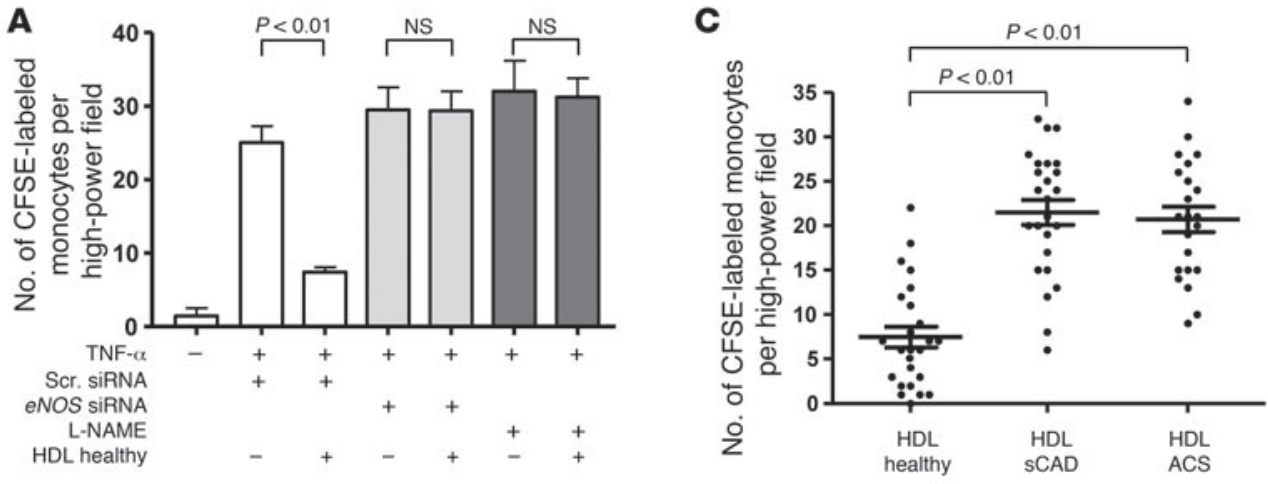

B TNF- $\alpha$

TNF- $\alpha+$ HDL healthy + scr. siRNA
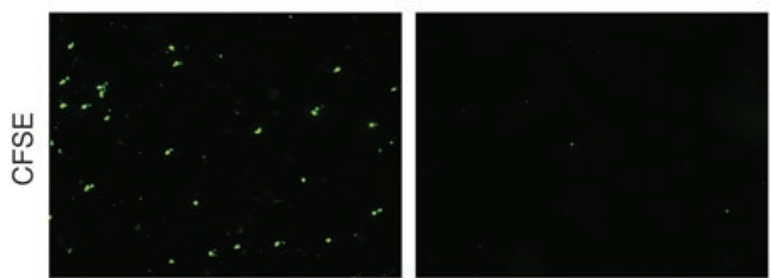

TNF- $\alpha+$ HDL healthy + eNOS SIRNA
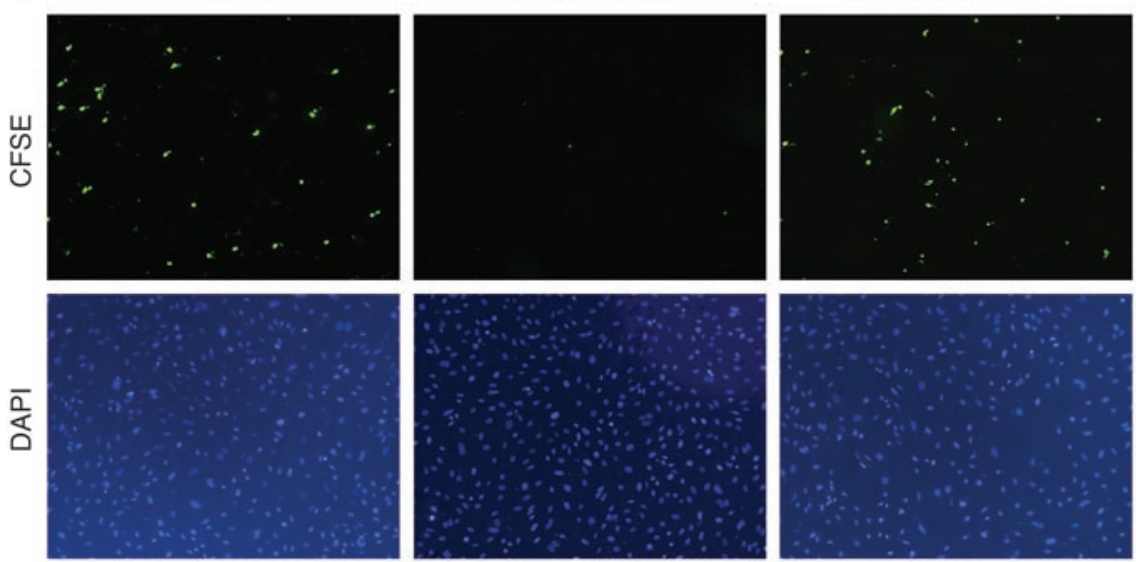

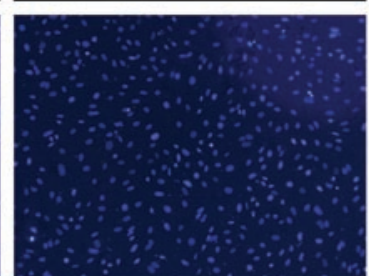

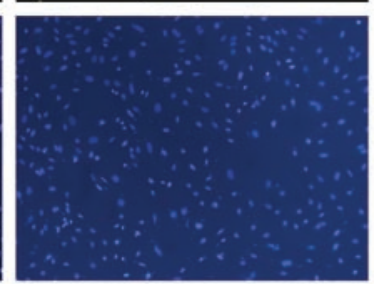

\section{Figure 4}

Role of eNOS for the effects of HDL on TNF- $\alpha$-stimulated endothelial monocyte adhesion. (A) Inhibition of eNOS-mediated NO production by siRNA-specific knockdown of eNOS or treatment with L-NAME $(1 \mathrm{mM})$ prevented the potent inhibitory effects of HDL from healthy subjects on endothelial cell monocyte adhesion ( $n=6-8$ per group). Endothelial cells were incubated with $\mathrm{HDL}(50 \mu \mathrm{g} / \mathrm{ml})$ from healthy subjects, and binding of CFSElabeled human monocytes was analyzed after 3 hours by fluorescence microscopy (HAECs were stimulated with TNF- $\alpha ; 5 \mathrm{ng} / \mathrm{ml}$ ). (B) Representative photographs (original magnification, $\times 10$ ) of endothelial monocyte adhesion obtained by fluorescence microscopy are shown. (C) Effects of $\mathrm{HDL}$ isolated from patients with SCAD and ACS as compared with HDL from healthy subjects on TNF- $\alpha-$ stimulated endothelial monocyte adhesion ( $n=25$ per group; data points for all study participants are shown). induced endothelial VCAM-1 expression (Figure 3E). In contrast to HDL $_{\text {Healthy, }}$ HDL $_{\mathrm{CAD}}$ increased VCAM-1 expression in unstimulated endothelial cells (Supplemental Figure 4B). Moreover, the inhibitory effects of $\mathrm{HDL}_{\mathrm{Healthy}}$ on endothelial monocyte adhesion were prevented by either eNOS siRNA interference or pharmacological inhibition of endothelial NO production (Figure 4, A and B). In line with the findings for NF- $\mathrm{KB}$ activation and VCAM-1 expression, HDL $_{C A D}$ did not inhibit TNF- $\alpha$-induced endothelial monocyte adhesion (Figure 4C) and stimulated endothelial monocyte adhesion under basal conditions (Supplemental Figure 4C).

Effects of HDL on endothelial repair in vivo. More recently, it has been suggested that HDL from healthy subjects promotes endothelial repair $(6,11,12,36)$. We therefore compared the effects of $\mathrm{HDL}_{\mathrm{CAD}}$ and $\mathrm{HDL}_{\text {Healthy }}$ on in vivo endothelial repair in a nude mouse model with carotid artery injury $(36,42,43)$. Administration of $\mathrm{HDL}_{\text {Healthy }}$ accelerated endothelial repair responses within 3 days after carotid injury (Figure 5, A and B). However, in nude mice, treatment with $\mathrm{HDL}_{\mathrm{CAD}}$ compared with buffer failed to significantly promote endothelial repair (Figure 5, A and B), indicating that HDL from patients with CAD lacks the capacity to stimulate endothelial repair. To investigate whether the effects of HDL on endothelial repair are dependent on endothelial NO synthase, we injected HDL $\mathrm{Healthy}_{\text {into }}$ eNOS-deficient mice. Notably, HDL $\mathrm{Healthy}_{\mathrm{H}}$ failed to stimulate endothelial repair in eNOS-deficient mice (Supplemental Figure 5, A and B), suggesting that eNOS plays a critical role for the stimulation of endothelial repair by HDL.

Specific binding of $H D L_{\text {Healthy }}$ and $H D L_{C A D}$ to endothelial cells - role of SR-BI. Specific endothelial binding of $\mathrm{HDL}_{\mathrm{CAD}}$ was moderately reduced as compared with $\mathrm{HDL}_{\mathrm{Healthy}}$ (Figure $5 \mathrm{C}$ ). In previous studies, it has been shown that endothelial SR-BI contributes to both endothelial binding of HDL (44) and stimulation of endothelial cell NO synthase by HDL, involving HDL-dependent cholesterol efflux $(7,8,16,17)$. We therefore compared the effects of endothelial SR-BI knockdown by RNA interference on the specific endothelial binding of $\mathrm{HDL}_{\mathrm{CAD}}$ and $\mathrm{HDL}_{\mathrm{Healthy}}$. Notably, SR-BI knockdown in endothelial cells reduced the specific endothelial binding of $\mathrm{HDL}_{\text {Healthy }}$ and $\mathrm{HDL}_{\mathrm{CAD}}$ to a similar extent (Figure 5D), a finding compatible with the concept that SR-BI-dependent specific endothelial binding of HDL is not significantly impaired in patients with CAD.

Effects of $H D L_{\text {Healthy }}$ and $H D L_{C A D}$ on macrophage cholesterol efflux. To determine whether the loss of the capacity of circulating $\mathrm{HDL}_{\mathrm{CAD}}$ to stimulate endothelial NO production is associated with changes in the capacity of HDL to stimulate cholesterol efflux, we further compared the effects of $\mathrm{HDL}_{\mathrm{Heal}}$ and $\mathrm{HDL}_{\mathrm{CAD}}$ on macrophage cholesterol efflux in vitro. Total and ABCA1-dependent cholesterol efflux was characterized in unstimulated and cAMP-stimulated J774 macrophages, respectively, whereas ABCG1-dependent cholesterol efflux was measured in HEK293 cells transfected with an ABCG1-expressing plasmid. In these studies we did not observe a significant difference in the capacity of circulating $\mathrm{HDL}_{\mathrm{Heal}}$ and HDL $_{\text {CAD }}$ to stimulate total, ABCA1-, or ABCG1-dependent cholesterol efflux (Supplemental Figure 6, A-C).

Effects of $H D L_{C A D}$ and $H D L_{\text {Healthy }}$ on endothelial PKC $\beta I I$ phosphorylation and its impact on eNOS-activating pathways - role of endothelial LOX-1 receptor. Our observation that HDL from patients with CAD 
A
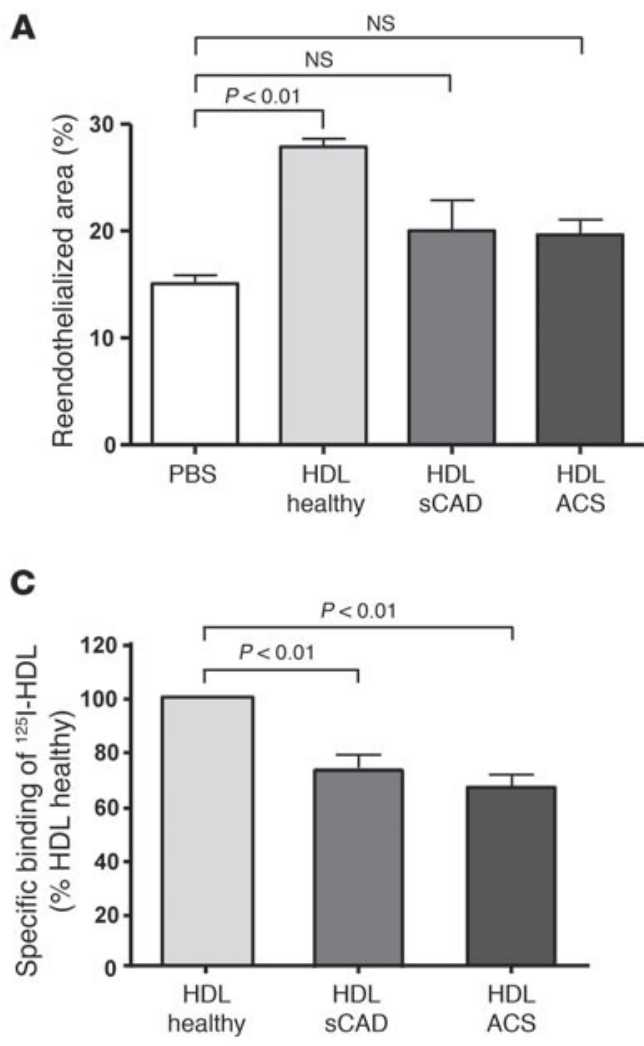

B
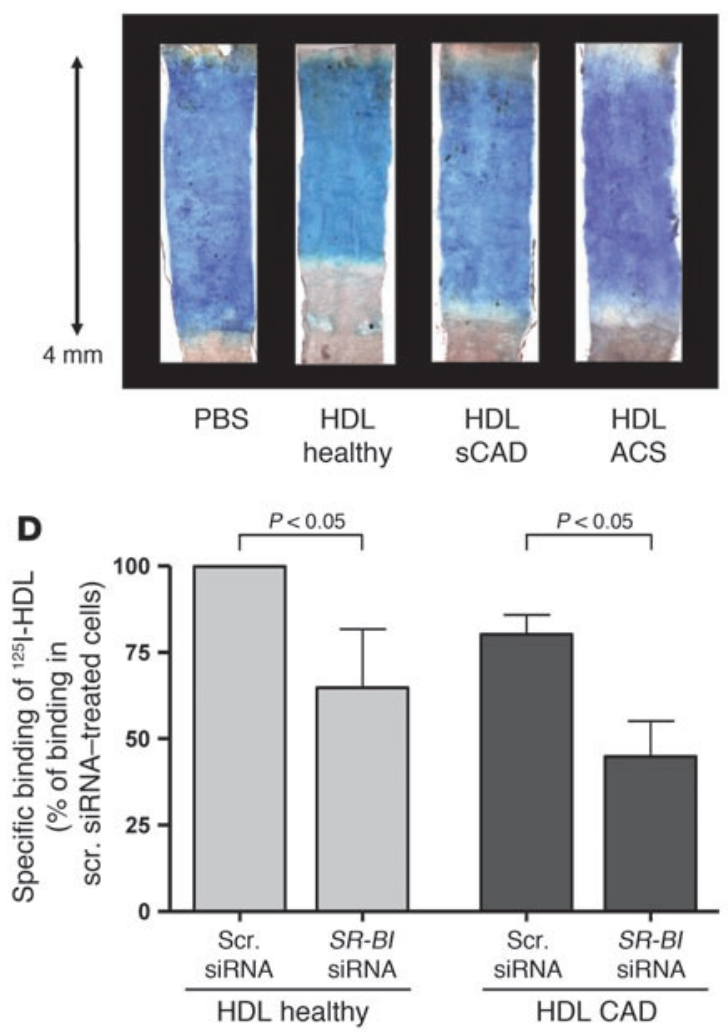

Figure 5

Impaired endothelial repair capacity and specific endothelial binding of HDL from patients with SCAD or ACS as compared with healthy subjects. (A) HDL from healthy subjects and patients with SCAD or ACS was injected in nude mice following carotid artery injury, and re-endothelialized area was determined after 3 days by staining with Evan's blue ( $n=6-8$ per group). (B) Representative photographs of carotid arteries from nude mice stained with Evan's blue. (C) HDL from healthy subjects and patients with SCAD or ACS was labeled with sodium iodide I-125, and binding of $125 \mathrm{I}-\mathrm{HDL}$ to endothelial cells was examined ( $n=8-10$ per group). (D) Effect of siRNA-specific knockdown of SR-BI on endothelial binding of HDL from healthy subjects and patients with CAD ( $n=4-5$ per group).

failed to stimulate endothelial NO production despite a preserved SR-BI-dependent endothelial binding of HDL $\mathrm{CAD}_{\mathrm{D}}$ suggested that $\mathrm{HDL}_{\mathrm{CAD}}$ may activate endothelial pathways inhibiting eNOS activation. Of note, endothelial PKC $\beta$ activation has been suggested to negatively impact endothelial function and to inhibit eNOSactivating pathways (45-48).

We therefore compared the effects of $\mathrm{HDL}_{\mathrm{CAD}}$ and HDL $\mathrm{Healthy}_{\mathrm{H}}$ on endothelial PKC $\beta$ II phosphorylation and membrane translocation, and the role of PKC $\beta$ II in the altered effects of $\mathrm{HDL}_{\mathrm{CAD}}$ on eNOS-activating pathways and endothelial NO production. Endothelial activation of PKC $\beta$ II was reduced by $\mathrm{HDL}_{\text {Healthy, as }}$ indicated by decreased PKC $\beta$ II phosphorylation at Ser660 (Figure $6 \mathrm{~A}$ ), which is known to be critical for PKC $\beta$ II-mediated endothelial proinflammatory effects (49).

In contrast, $\mathrm{HDL}_{\mathrm{CAD}}$ increased endothelial PKC $\beta \mathrm{II}$ phosphorylation at Ser660 as compared with buffer treatment (Figure 6A) and induced membrane translocation of PKC $\beta \mathrm{II}$ in endothelial cells (Figure 6B), suggesting activation of PKC $\beta$ II by HDL $\mathrm{CAD}_{\mathrm{C}}$. Of note, LY379196, a nonselective inhibitor of PKC $\beta$ I and PKC $\beta I$ is isoforms, as well as CGP53353, a highly selective inhibitor of PKC $\beta I I$, partially restored the impaired capacity of $\mathrm{HDL}_{\mathrm{CAD}}$ to stimulate endothelial NO production (Figure 6C), Akt phosphorylation at
Ser473 (Supplemental Figure 7), and Akt-dependent eNOS phosphorylation at Ser1177 (Figure 6D).

The endothelial multiligand receptor LOX-1 has been implicated in the binding of oxidized lipoproteins and the activation of endothelial PKC $\beta(50,51)$. Notably, inhibition of the endothelial LOX-1 receptor with a blocking antibody prevented the increase in PKC $\beta I I$ phosphorylation at Ser660 in response to $\mathrm{HDL}_{\mathrm{CAD}}$ (Figure $6 \mathrm{E})$, suggesting that $\mathrm{HDL}_{\mathrm{CAD}}$ activates $\mathrm{PKC} \beta \mathrm{II}$, at least in part, by interacting with the endothelial LOX-1 receptor. In line with these findings, inhibition of the endothelial LOX-1 receptor resulted in an increased endothelial NO production in endothelial cells treated with $\mathrm{HDL}_{\mathrm{CAD}}$ but not $\mathrm{HDL}_{\text {Healthy }}$ (Figure 6F). Together, these findings suggest that LOX-1-dependent activation of PKC $\beta I$ leads to inhibition of eNOS-activating pathways and impaired stimulation of endothelial NO production by $\mathrm{HDL}_{\mathrm{CAD}}$.

$M D A$ content of $H D L$ and its role in $H D L$-mediated stimulation of endothelial NO production. The endothelial LOX-1 receptor is activated by oxidized lipoproteins $(50,51)$. We have therefore examined the potential role of lipid peroxidation, in particular the formation of malondialdehyde (MDA; a product of polyunsaturated fatty acid peroxidation), in the effects of HDL on endothelial NO production. 

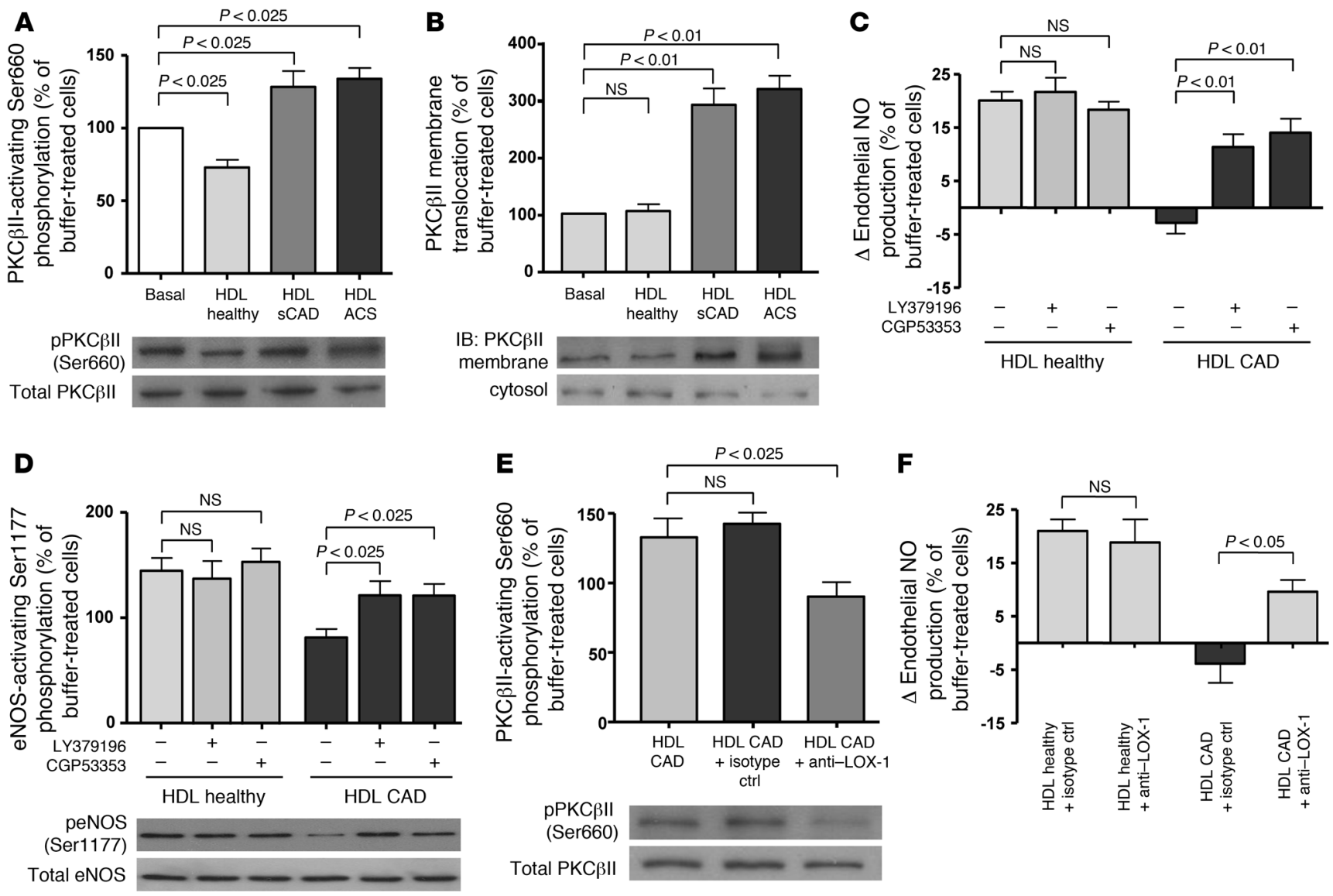

Figure 6

Differential effects of HDL from healthy subjects and patients with SCAD or ACS on endothelial LOX-1 and PKC $\beta$ II activation - role of PKC $\beta$ II activation in the altered effects of $\mathrm{HDL}$ on endothelial Akt and eNOS-activating/inhibiting phosphorylation in patients with $\mathrm{CAD}$. (A) Effect of HDL from healthy subjects and patients with SCAD and ACS on PKC $\beta I$-activating phosphorylation at Ser660 and (B) membrane translocation of PKC $\beta$ II in endothelial cells ( $n=8-12$ per group). (C) Incubation of endothelial cells with the nonselective inhibitor of PKC $\beta$ I and PKC $\beta I I$ isoforms LY379196 and CGP53353, a highly selective inhibitor of PKC $\beta$ II, restored the ability of HDL from patients with CAD to stimulate endothelial NO production and (D) the activating eNOS phosphorylation at Ser1177 ( $n=8-10$ per group). (E) Pretreatment of endothelial cells with an anti-LOX-1 blocking antibody prevented the increase in PKC $\beta$ II phosphorylation at Ser660 ( $n=6-8$ per group). (F) Moreover, incubation of endothelial cells with an anti-LOX-1 blocking antibody improved the capacity of HDL from patients with CAD, but not from healthy subjects, to stimulate endothelial NO production ( $n=8-10$ per group).

Notably, the protein-bound MDA content was increased in $\mathrm{HDL}_{\mathrm{CAD}}$ as compared with $\mathrm{HDL}_{\text {Healthy }}$ (Figure $7 \mathrm{~A}$ ), and mass spectrometry analyses revealed increased MDA modification of lysine residues in $\mathrm{HDL}_{\mathrm{CAD}}$ (Figure 7B). To determine whether modification of HDL by MDA can modulate the capacity of HDL to stimulate endothelial NO production, we incubated $\mathrm{HDL}_{\text {Healthy }}$ with increasing amounts of freshly prepared MDA and measured the effects of the modified HDL on endothelial NO production. Of note, MDA modification of $\mathrm{HDL}_{\mathrm{Healthy}}$ resulted in a dose-dependent increase in the amount of MDA in HDL (control without addition of MDA: $0.31 \pm 0.04$ nmol MDA/mg HDL; after addition of $12.5 \mathrm{~mol} \mathrm{MDA} / \mathrm{mol}$ apoA-I: $0.99 \pm 0.05 \mathrm{nmol} \mathrm{MDA} / \mathrm{mg}$ HDL,$P<0.01$ vs. control; after addition of $25 \mathrm{~mol} \mathrm{MDA} / \mathrm{mol}$ apoA-I: $1.31 \pm 0.14 \mathrm{nmol} \mathrm{MDA} / \mathrm{mg} \mathrm{HDL}$, $P<0.01$ vs. control) and a dose-dependent reduction in the capacity of HDL to stimulate endothelial NO production (Figure 7C). Inhibition of the endothelial LOX-1 receptor with a blocking antibody partially prevented the impairment of the effects of MDA-modified HDL on endothelial NO production (Figure 7D). Notably, the amount of pro- tein-bound MDA or MDA-lysine adducts in HDL modified by 12.5 mol MDA/mol apoA-I - a concentration that resulted in a significant reduction in the capacity of $\mathrm{HDL}_{\text {Healthy }}$ to stimulate endothelial NO production (Figure 7C) - was similar to the amount of MDA observed in $\mathrm{HDL}_{\mathrm{CAD}}$ (Figure 7, A and B, and Supplemental Figure 8, A-C). These findings suggest that MDA modification of HDL represents a potential pathway contributing to the reduced capacity of HDL $_{\text {CAD }}$ to stimulate endothelial NO production.

Moreover, MDA-modified HDL increased activation of endothelial PKC $\beta I I$, as detected by phosphorylation of PKC $\beta I I$ at Ser660, and the stimulation of endothelial PKC $\beta I I$ by MDA-modified HDL was prevented after inhibition of endothelial LOX-1 with a specific blocking antibody (Figure 7E), suggesting that the adverse effects of MDA-modified HDL on endothelial NO production are, at least in part, mediated via LOX-1-dependent activation of PKC 3 II. Notably, HDL-associated PON1 has been reported to prevent MDA formation in HDL (52). Moreover, we have observed a relationship between reduced PON1 activity and endothelial dys- 
A

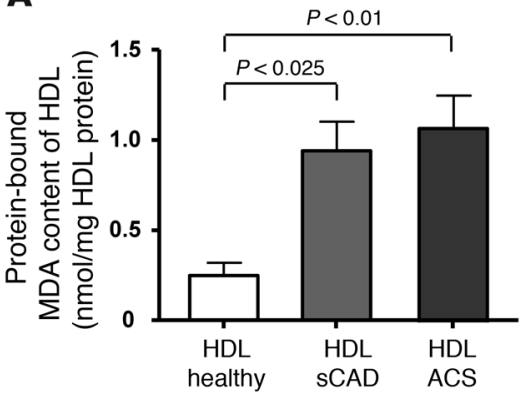

D

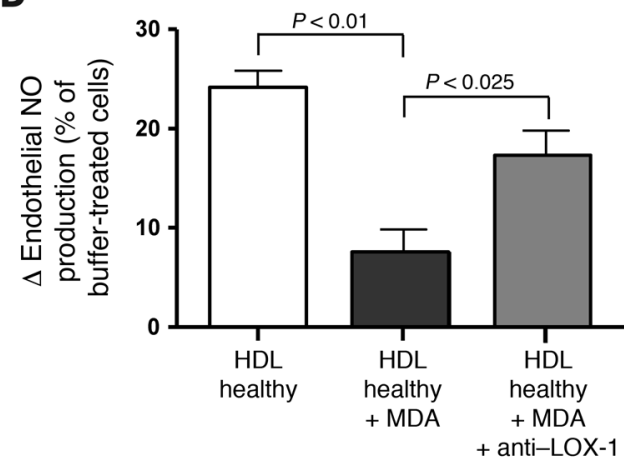

B

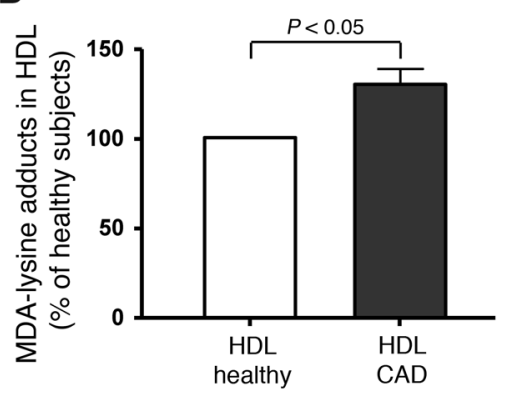

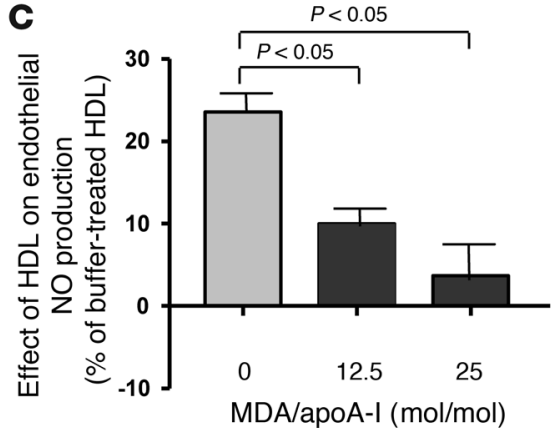

MDA/apoA-I ( $\mathrm{mol} / \mathrm{mol})$

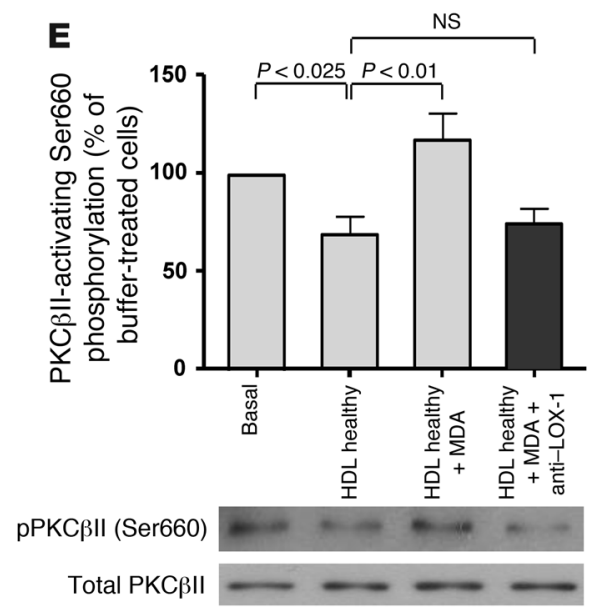

Figure 7

Critical role of HDL-associated MDA in the impaired capacity of HDL to increase endothelial NO production and in the increased stimulation of PKC $\beta$ II by HDL. (A) Protein-bound MDA content in HDL from healthy subjects and patients with sCAD or ACS was measured by spectrophotometry, as described in more detail in Methods ( $n=12-14$ per group). (B) Mass spectrometry analysis of MDA-lysine adducts in HDL from healthy subjects and patients with CAD ( $n=4$ per group). (C) Effect of MDA-modified HDL on endothelial NO production, as measured by ESR spectroscopy ( $n=8$ per group). (D) Inhibition of endothelial LOX-1 with a specific blocking antibody partially prevented the decrease in endothelial NO production in response to MDA-modified HDL (12.5 mol MDA $/ \mathrm{mol}$ apoA-I; $n=6$ per group). (E) Modification of HDL by MDA (12.5 mol MDA/mol apoA-I; $n=6$ per group) resulted in an increased activation of endothelial PKC $\beta I$ that was prevented by a specific blocking antibody against endothelial LOX-1 ( $n=6-10$ per group).

function in patients with APS (37). We therefore examined whether an alteration of PON1 activity may play a causal role in the impaired capacity of $\mathrm{HDL}_{\mathrm{CAD}}$ to stimulate endothelial NO production.

Role of PON1 in HDL-mediated activation of PKC $\beta I$ II and stimulation of endothelial NO production. PON1 activity was substantially reduced in $\mathrm{HDL}_{\mathrm{CAD}}$ when compared with $\mathrm{HDL}_{\mathrm{Healthy}}$, as quantified by either paraoxonase or arylesterase activity (Figure 8, A and B). In contrast, the content of PON1 was increased in $\mathrm{HDL}_{\mathrm{CAD}}$ as compared with $\mathrm{HDL}_{\text {Healthy }}$ (Figure $8 \mathrm{C}$ ), suggesting an inactivation of PON1 in $\mathrm{HDL}_{\mathrm{CAD}}$. Interestingly, extended-release niacin therapy was associated with significantly increased HDL-associated PON1 activity (Supplemental Figure 9), indicating that inactivation of PON1 may, at least in part, be prevented by this treatment.

To further determine the functional relevance of HDL-associated PON1 activity for the endothelial effects of HDL, we examined the effects of PON1 inhibition by either hydroxyquinoline or EDTA $(53,54)$ on endothelial PKC $\beta I$ II activation and eNOS-activating pathways as well as endothelial NO production. Paraoxonase activity measurements revealed effective inhibition of PON1 activity after incubation of $\mathrm{HDL}_{\text {Healthy }}$ with hydroxyquinoline $(78 \% \pm 9 \%$,
$P<0.01)$ or EDTA $(71 \% \pm 12 \%, P<0.01)$. Notably, after inhibition of HDL-associated PON1 activity, $\mathrm{HDL}_{\text {Healthy }}$ gained the ability to phosphorylate endothelial PKC $\beta I$ at Ser660 (Figure 8F). HDL $\mathrm{Healthy}_{\mathrm{H}}$ with inhibited PON1 activity did not stimulate eNOS-activating phosphorylation at Ser1177 (Figure 9A) and caused a marked upregulation of eNOS-inhibiting phosphorylation at Thr495 (Figure 9B). Moreover, inhibition of PON1 activity in $\mathrm{HDL}_{\text {Healthy }}$ by hydroxyquinoline and EDTA resulted in a loss of the capacity of HDL to stimulate endothelial NO production (Figure 9C), whereas stimulation of endothelial NO production by bradykinin was not affected by either PON1 inhibitor (Supplemental Figure 10). Taken together, these findings suggest that HDL-associated PON1 activity is critical for the eNOS-stimulating properties of HDL. In line with these findings and the interpretation that HDL-associated PON1 is a determinant for the capacity of HDL to stimulate endothelialprotective effects, inhibition of PON1 prevented the effects of HDL $_{\text {Healthy }}$ on TNF- $\alpha$-stimulated endothelial VCAM- 1 expression (Supplemental Figure 11), endothelial monocyte adhesion (Figure 9D), and endothelial repair (Figure 9E). In addition, supplementation of HDL from patients with CAD with purified PON1 partially 


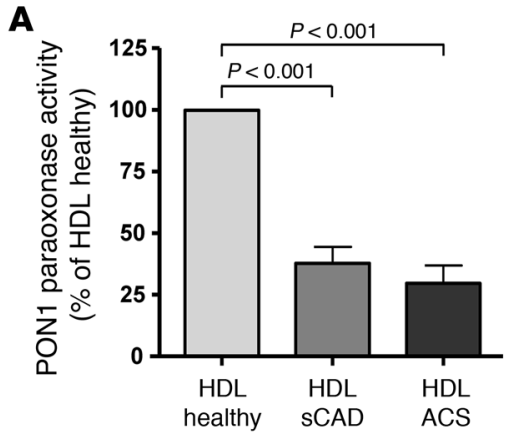

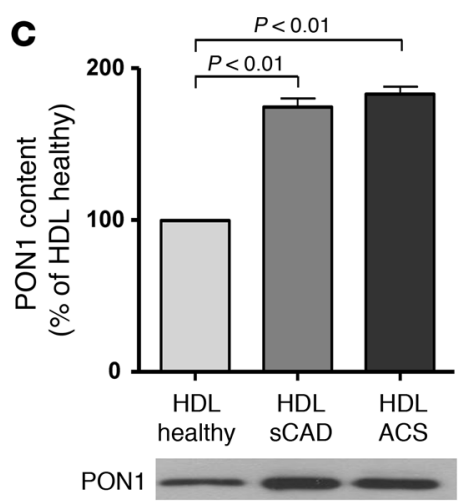
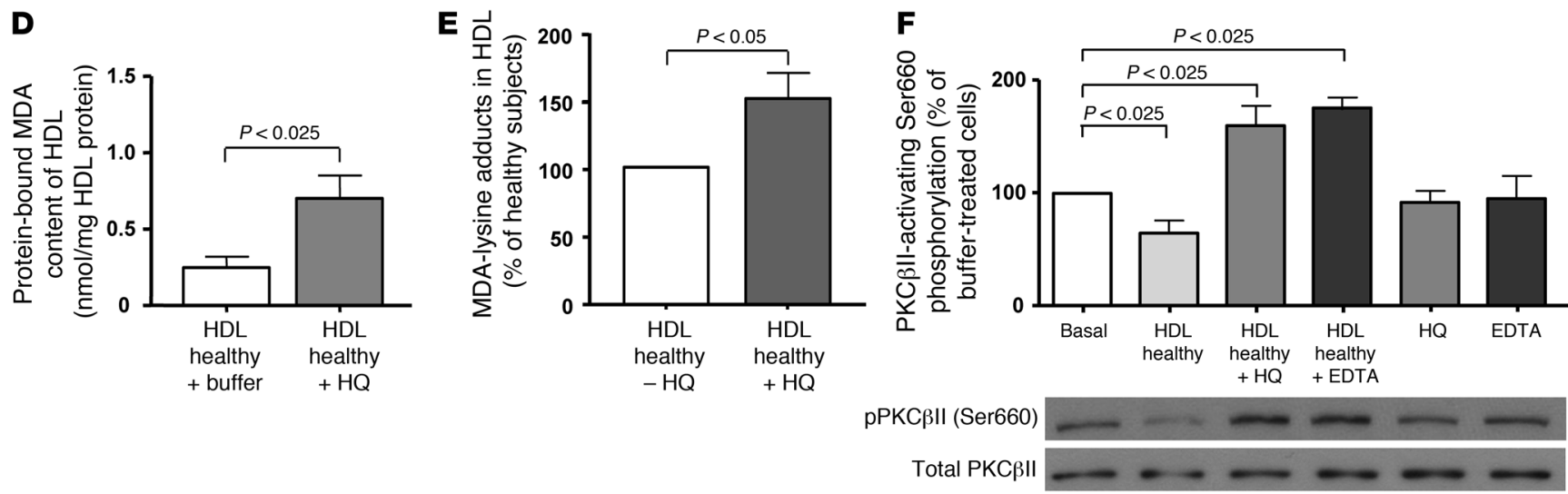

Figure 8

Activity and content of HDL-associated PON1 in healthy subjects and patients with SCAD or ACS - effect of inhibition of PON1 on HDL-associated MDA content and phosphorylation of PKC $\beta$ II at Ser660. (A) Paraoxonase and (B) arylesterase activities of HDL-associated PON1 isolated from healthy subjects and patients with SCAD or ACS, as measured by UV spectrophotometry ( $n=25$ per group). (C) PON1 content in HDL isolated from healthy subjects and patients with SCAD or ACS was determined by Western blot analysis ( $n=25$ per group). (D) Effect of the specific PON1 inhibitor hydroxyquinoline (HQ, $200 \mu \mathrm{M})$ on protein-bound MDA content and (E) MDA-lysine adducts in HDL, as detected by spectrophotometry and mass-spectrometry analysis, respectively $(n=4-6$ per group). (F) Effect of HDL pretreated with the PON1 inhibitors HQ or EDTA ( $5 \mathrm{mM}$ ) on phosphorylation of PKC $\beta$ II at Ser660 was detected by Western blot analysis ( $n=8-10$ per group).

improved the capacity of HDL to stimulate endothelial NO production under physiological conditions (i.e., $37^{\circ} \mathrm{C}$; Figure 9F), supporting the concept that HDL-associated PON1 activity impacts on the effects of HDL on endothelial NO production.

To further examine the effects of PON1 on protein-bound MDA content in HDL, we inhibited HDL-associated PON1 activity by either hydroxyquinoline or EDTA. Importantly, inhibition of HDLassociated PON1 activity increased both the protein-bound MDA content and the amount of MDA-lysine adducts in HDL $\mathrm{H}_{\text {Healthy }}$ under physiological conditions $\left(37^{\circ} \mathrm{C}\right)$ as used in the endothelial bioassays (Figure 8, D and E). Notably, accumulation of MDA in $\mathrm{HDL}_{\text {Healthy }}$ was only observed after inhibition of PON1 activity under physiological conditions (Supplemental Figure 8C). Supplementation of MDA-modified HDL with purified PON1 did not significantly reduce the protein-bound MDA content or MDA-lysine adducts in HDL (Supplemental Figure 8, A and B). These findings suggest that PON1 prevents MDA formation in HDL under physiological conditions, rather than directly degrading MDA.

Moreover, in $\mathrm{HDL}_{\mathrm{CAD}}$ an accumulation of MDA was observed under physiological conditions that was prevented by supplementation of $\mathrm{HDL}_{\mathrm{CAD}}$ with purified PON1 (Supplemental Figure 8C), further supporting the concept that PON1 prevents formation of MDA in HDL. These observations may also, in part, explain why we have observed a partial effect of PON1 supplementation of $\mathrm{HDL}_{\mathrm{CAD}}$ on its capacity to stimulate endothelial cell NO production (Figure 9F).

Effect of HDL from Pon1-deficient mice on endothelial NO production, endothelial superoxide production, and endothelial inflammatory activation. To further examine the role of PON1 in the effects of HDL on endothelial NO production on a molecular level, we isolated HDL from Pon1-deficient and wild-type mice. Of note, HDL from Pon1-deficient mice failed to stimulate NO production in mouse aortic endothelial cells (Figure 10A) and did not inhibit basal and TNF- $\alpha$-stimulated superoxide production (Figure 10B). Furthermore, HDL from Pon1-deficient mice failed to inhibit endothelial VCAM-1 expression (Figure 10C) and endothelial monocyte adhesion (Figure 10D), supporting the concept that PON1 is a regulator of the capacity of HDL to exert endothelial-vasoprotective effects. Notably, supplementation of HDL from Pon1-deficient mice with purified PON1 partially improved the capacity of HDL to stimulate endothelial NO production (Figure 10E).

\section{Discussion}

Low HDL cholesterol levels are associated with an increased risk of CAD and major cardiovascular events $(1,2)$. Besides promotion of 

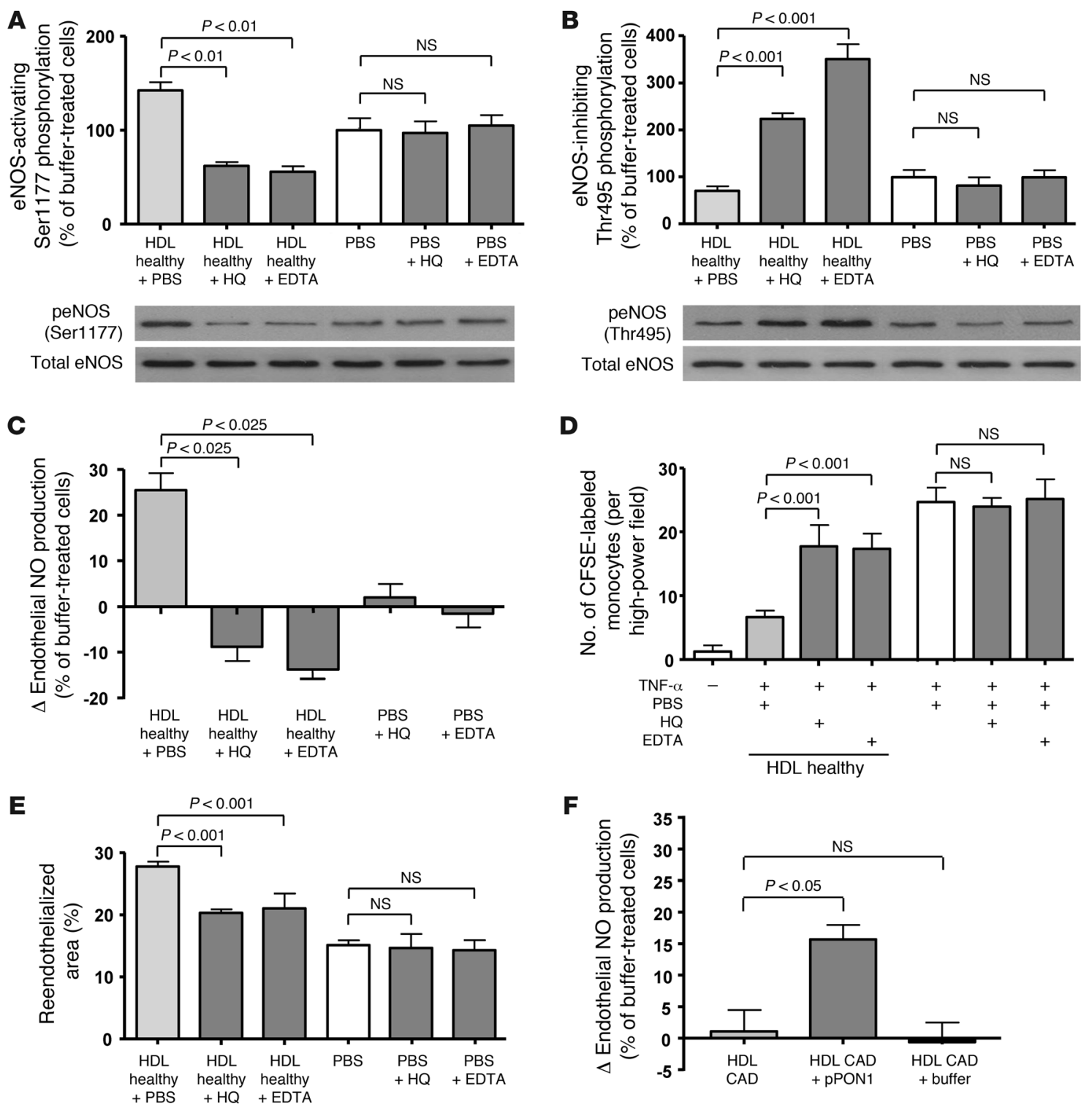

\section{Figure 9}

Effect of inhibition of HDL-associated PON1 on eNOS-activating signaling pathways, endothelial cell NO production, endothelial cell inflammatory activation, and endothelial repair. Effect of supplementation of HDL from CAD patients with recombinant PON1 on the capacity of HDL to stimulate endothelial NO production. (A) HDL-associated PON1 was inhibited either by hydroxyquinoline (200 $\mu$ M) or EDTA (5 mM), and the effect of HDL on the activating eNOS phosphorylation at Ser1177 and (B) the inhibitory eNOS phosphorylation at Thr495 was examined by Western blot analysis ( $n=6-7$ per group). (C) Inhibition of HDL-associated PON1 resulted in a loss of the capacity of HDL to stimulate endothelial NO production, as measured by ESR spectroscopy ( $n=6-8$ per group). (D) Furthermore, treatment of HDL from healthy subjects with PON1 inhibitors impaired the capacity of HDL to inhibit TNF- $\alpha$-stimulated endothelial monocyte adhesion ( $n=8-10$ per group). (E) Importantly, inhibition of PON1 in HDL from healthy subjects prevented stimulation of endothelial repair after carotid artery injury in nude mice ( $n=6$ per group). (F) Supplementation of HDL from patients with CAD with purified PON1 (pPON1; $5 \mathrm{U} / 100 \mu \mathrm{g}$ HDL protein) partially restored the capacity of HDL to stimulate endothelial NO production as measured by ESR spectroscopy ( $n=6-9$ per group).

cholesterol efflux from macrophage foam cells $(3,4)$, stimulation of eNOS-dependent NO production $(7-10,18,21,36)$ and mediation of endothelial repair mechanisms (11-15), as well as antiinflammatory effects (55), have been proposed as potential antiatherogenic properties of HDL. HDL-raising therapies are currently being intensely evaluated for prevention and treatment of CAD.

The present study demonstrates that HDL from patients with either SCAD or an ACS - in contrast to HDL from healthy sub- jects - fails to stimulate endothelial NO production. Of note, reduced endothelial NO bioavailability is thought to contribute to development and progression of atherosclerosis, at least in part by promoting endothelial inflammatory activation (22, 23). In this respect, the inability of HDL from patients with CAD to stimulate endothelial NO production, as observed in the present study, suggests a loss of this potential antiatherogenic property of HDL (22). 
A

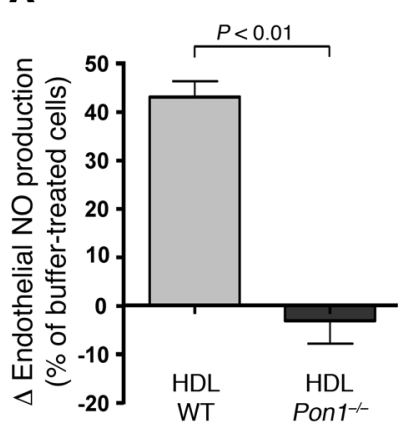

B

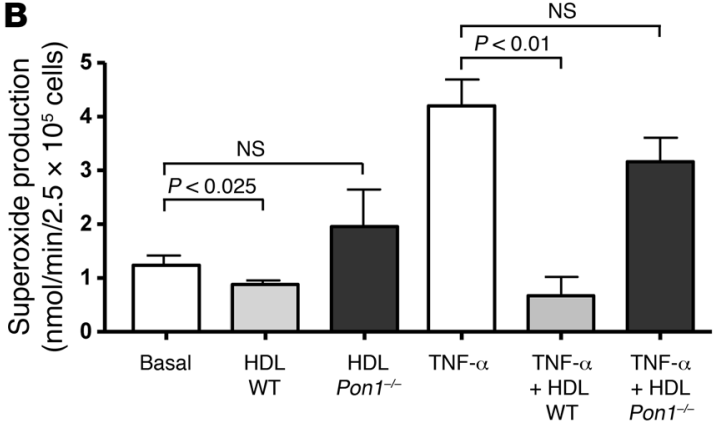

$\mathbf{E}$
C

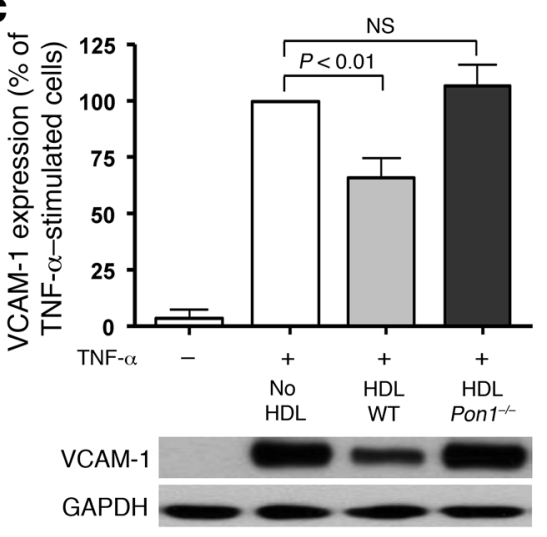

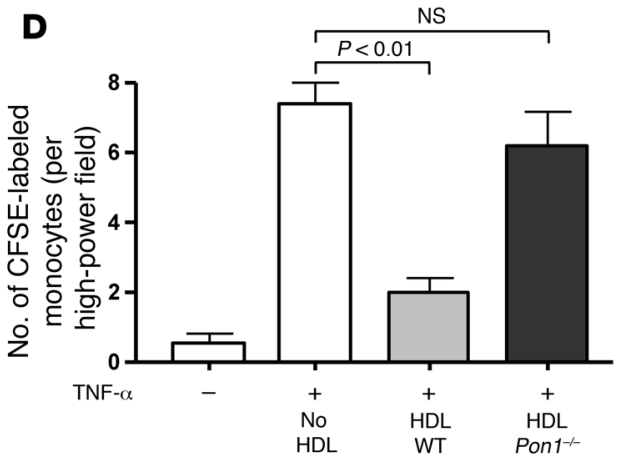

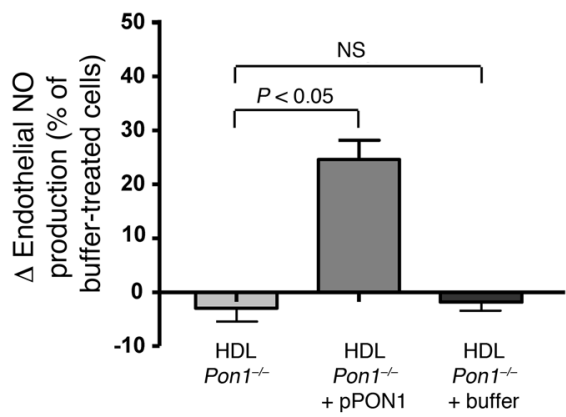

Figure 10

Effects of HDL isolated from Pon1-deficient mice or their wild-type littermates on endothelial cell NO production, superoxide production, and endothelial inflammatory activation. (A) In contrast to HDL from wild-type littermates, HDL isolated from Pon1-deficient mice inhibited rather than stimulated NO production in mouse aortic endothelial cells, as detected by ESR spectroscopy analysis ( $n=5$ per group). (B) In addition, HDL from Pon1-deficient mice failed to inhibit basal and TNF- $\alpha$-stimulated superoxide production in mouse aortic endothelial cells (ESR spectroscopy analysis, $n=5-7$ per group). (C) Whereas HDL from wild-type littermates potently inhibited endothelial inflammatory activation, HDL from Pon1-deficient mice did not inhibit TNF- $\alpha$-stimulated VCAM-1 expression and (D) endothelial monocyte adhesion ( $n=5-6$ per group). (E) Supplementation of HDL from Pon1-deficient mice with purified PON1 (5 U/100 $\mu \mathrm{g} \mathrm{HDL}$ protein) partially restored the capacity of HDL to stimulate endothelial NO production as measured by ESR spectroscopy ( $n=5-6$ per group).

Our findings suggest that stimulation of endothelial NO production is important for HDL's ability to inhibit inflamma-

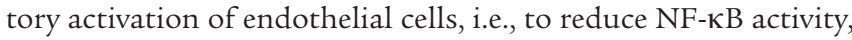
VCAM-1 expression, and monocyte adhesion, which are thought to play a role in the development and progression of atherosclerosis $(56,57)$. In addition, HDL-induced stimulation of endothelial repair was not observed in eNOS-deficient mice, suggesting a role of eNOS in this process. We therefore examined the mechanisms underlying alterations of these potential endothelial-atheroprotective effects of HDL in patients with CAD.

Endothelial superoxide production, which may limit NO bioavailability (39), was not increased in response to $\mathrm{HDL}_{\mathrm{CAD}}$, suggesting that the altered endothelial $\mathrm{NO}$ response resulted from an impaired capacity of HDL $\mathrm{CAD}_{\text {to }}$ stimulate eNOS activation rather than from a stimulation of endothelial superoxide production. In previous studies it has been shown that eNOS activation in response to HDL is mediated via Akt-dependent eNOS phosphorylation at Ser1177 (8). Notably, in the present study we have observed that $\mathrm{HDL}_{\mathrm{CAD}}$ in contrast to $\mathrm{HDL}_{\text {Healthy }}$ activates endothelial PKC $\beta I$ I, leading to inhibition of Akt-dependent eNOSactivating phosphorylation at Ser1177 and increased phosphorylation of eNOS at Thr495, which inhibits eNOS activity. Our findings further indicated that increased endothelial PKC $\beta I$ activation by $\mathrm{HDL}_{\mathrm{CAD}}$ was involved in the impaired effects of $\mathrm{HDL}_{\mathrm{CAD}}$ on eNOS-derived NO production and was mediated, at least in part, via the endothelial LOX-1 receptor. Endothelial PKC $\beta$ activation has been observed to promote endothelial dysfunction, to inhibit Akt-dependent eNOS-activating phosphorylation at Ser1177, to increase eNOS-inhibiting phosphorylation at Thr495 $(45-48,58)$, and to promote endothelial inflammatory activation (49).

Endothelial SR-BI mediates specific endothelial binding of HDL (44) and stimulates pathways leading to the activation of eNOS in response to the lipoprotein $(7,8,11,17)$. In the present study we observed that knockdown of endothelial SR-BI by RNA interference reduced specific binding of HDL from both healthy subjects and patients with CAD to a similar extent. These findings are compatible with the notion that the interaction of HDL with SR-BI for endothelial binding of HDL is not significantly impaired in patients with CAD. Moreover, recent studies have observed that HDL-mediated cholesterol efflux contributes to eNOS activation in response to $\operatorname{HDL}(10,16,18)$. In the present study we did not observe a significant difference in the macrophage cholesterol efflux capacity of circulating HDL from patients with CAD and healthy subjects. These findings are compatible with the concept that the impaired capacity of circulating HDL to stimulate endothelial NO production is related to endothelial LOX-1- 
dependent PKC $\beta$ II activation rather than to a major impairment in SR-BI binding or cholesterol efflux. This concept is further supported by the observation that the capacity of $\mathrm{HDL}_{\mathrm{CAD}}$ to stimulate endothelial eNOS-activating pathways and endothelial NO production could be restored, at least in part, by either endothelial LOX-1 blockade or endothelial PKC $\beta$ II inhibition.

It is likely that the impairment of the macrophage cholesterol efflux capacity of HDL - which has been reported to occur after more extensive oxidative modification of HDL, for example, in atherosclerotic plaque $(32,33)-$ is a pathophysiological mechanism that is more operative within the atherosclerotic lesion. Indeed, HDL isolated from atherosclerotic plaques had an impaired capacity to stimulate macrophage cholesterol efflux $(32,33)$.

In addition, we aimed to identify alterations in HDL ${ }_{C A D}$ leading to the gain of endothelial LOX-1- and PKC $\beta$ II-activating properties and the subsequent inhibition of endothelial eNOS-activating pathways. In patients with CAD, the MDA content of HDL was increased, and modification of HDL $\mathrm{L}_{\text {Healthy }}$ with MDA impaired the capacity of HDL to stimulate endothelial NO production, and this was at least in part mediated via the endothelial LOX-1 receptor. Notably, PON1 has been observed to prevent MDA formation in HDL (52) and to promote HDL-mediated inactivation of oxidized lipids in $\operatorname{LDL}(52,59,60)$. In addition, oxidized LDL has been suggested to activate endothelial PKC $\beta$ (50). These observations raised the possibility that PON1 may be involved in the gain of endothelial LOX-1- and PKC $\beta$ II-activating properties of $\mathrm{HDL}_{\mathrm{CAD}}$. Furthermore, in a recent study in patients with APS, we observed a relationship between reduced serum PON1 activity and endothelial dysfunction (37). We therefore evaluated whether a reduced PON1 activity may have a causal role in the impaired effects of HDL on endothelial NO production.

In the present study, HDL-associated PON1 activity was markedly reduced in patients with $\mathrm{CAD}$, and we demonstrate that impaired HDL-associated PON1 activity is involved in the gain of endothelial PKC $\beta$ II-activating properties of HDL, leading to a subsequently impaired capacity to stimulate eNOS-activating pathways and endothelial NO production. Consistent with these findings, HDL isolated from Pon1-deficient mice failed to stimulate endothelial NO production. Furthermore, supplementation of HDL from patients with CAD or Pon1-deficient mice with purified PON1 partially improved the capacity of HDL to stimulate endothelial NO production. These findings suggest a role of HDL-associated PON1 activity in maintaining the endothelialatheroprotective effects of HDL, at least in part by preventing formation of the lipid peroxidation product MDA. Our studies are in line with the concept that PON1 prevents MDA formation in HDL rather than directly degrading MDA, which may explain why we observed only a partial effect of PON1 supplementation on the capacity of HDL $\mathrm{CAD}_{\mathrm{CA}}$ to stimulate endothelial NO production under physiological conditions. Furthermore, it is conceivable that PON1 also acts on other chemical entities in the HDL particle that impact its endothelial effects, which will need to be explored further in future studies.

Notably, in recent studies it has been observed that modification of reconstituted HDL (i.e., apoA-I and phospholipids) with myeloperoxidase leads to an impairment of its cholesterol efflux and antiinflammatory properties, likely due to a modification of apoA-I (61). Given that reconstituted HDL does not contain PON1, the question arises how this can be reconciled with a role of PON1 for the dysfunction of HDL. Importantly, however, a more recent study has described a critical role of apoA-I modification by MDA in the ability of apoA-I to promote cellular cholesterol efflux (33). These findings suggest that although PON1 prevents lipid oxidation of HDL and the subsequent formation of advanced lipid oxidation products, such as MDA, it thereby likely also prevents modifications of HDL-associated proteins such as apoA-I.

Moreover, in experimental studies PON1 has been observed to prevent atherosclerotic lesion development $(62,63)$, and in recent clinical studies reduced serum activity of paraoxonase was associated with increased cardiovascular risk $(64,65)$. The present study for the first time to our knowledge provides evidence that HDL-associated PON1 activity plays a role in maintaining the endothelial-atheroprotective effects of HDL, i.e., the capacity to stimulate endothelial NO production. However, the molecular mechanisms leading to PON1 inactivation in HDL from patients with $\mathrm{CAD}$ and its causal role in the development and progression of CAD need to be further examined in future studies. Notably, in this respect several studies have suggested a role of myeloperoxidase in modification of proteins in HDL, which has been shown in particular for apoA-I, but may potentially also apply to other HDL-associated proteins $(32,66)$.

Our findings raise the question of whether the impaired capacity of HDL from patients with CAD to stimulate eNOS-activating pathways and NO production may play a role in development and/ or progression of atherosclerosis or is a consequence of the disease process. As mentioned above, numerous studies have suggested that endothelial dysfunction, in particular reduced endothelial NO bioavailability, promotes atherosclerotic lesion development, and endothelial dysfunction has been shown to be a predictor of an increased risk of clinical cardiovascular events at later stages of the disease $(23,67)$. These observations raise the possibility that the altered effects of HDL on endothelial NO production may favor development and progression of atherosclerotic cardiovascular disease. However, the relationship between altered biological functions of HDL and endothelial dysfunction and cardiovascular risk will have to be characterized in future studies.

In the present study we examined the endothelial effects of HDL in bioassays using cultured endothelial cells. However, we have also observed stimulation of endothelial repair in vivo after arterial injury in mice receiving an injection of HDL from healthy subjects, but not after administration of HDL from patients with CAD. Moreover, these effects of HDL on endothelial repair in vivo were dependent on eNOS activation, since they were not observed in eNOS-deficient mice, suggesting that HDL also activates eNOSdependent endothelial responses in vivo. In addition, in a previous study we observed improved endothelium-dependent, NO-mediated vasodilation in patients with hypercholesterolemia after infusion of reconstituted HDL (19), further supporting the concept that HDL may activate eNOS-dependent NO production in vivo.

The influence of different non-HDL-targeted medications used in patients with CAD on the effects of HDL on eNOS-dependent pathways is largely unknown and needs to be studied in the future. In a recent study by Ansell et al. (35), it was suggested that the proinflammatory effects of HDL from patients with CAD were attenuated by statin therapy. However, in the present study we did not observe a significant difference in the effects on endothelial NO production between HDL from a subgroup of patients with and a subgroup without a certain medication (i.e., statins, beta blocker, and ACE-I/ARB). In fact, the direct proinflammatory properties of HDL from patients with CAD are likely not related 
to an eNOS-dependent mechanism, since they were also observed in the present study using HDL from patients with SCAD that did not result in a significant inhibition of endothelial NO production. The mechanisms underlying these proinflammatory properties of $\mathrm{HDL}_{\mathrm{CAD}}$ need to be further characterized in future studies. A recent study has suggested that the hemoglobin/haptoglobin pathway is involved in the formation of proinflammatory HDL in atherosclerotic mice and patients with CAD (68).

Notably, in a recent large clinical outcome trial testing the effects of the CETP inhibitor torcetrapib, which results in substantially elevated HDL cholesterol levels, a significant increase in cardiovascular events and in total mortality was observed (31). While off-target effects of the drug likely played a role, these findings also raise the possibility that the biological activity of on-treatment HDL in addition to its plasma levels need to be taken into account for the development of HDL-raising treatment strategies. The findings of the present study further support the notion that not only the capacity of HDL-targeted treatment strategies to raise HDL plasma levels, but also their effects on vasoprotective properties of HDL, need to be considered. It is likely that HDL-raising therapeutic strategies are more likely to result in cardiovascular protection when the HDL in treated patients itself exerts vasoprotective effects.

In summary, the present study provides evidence that HDL of patients with either SCAD or ACS fails to stimulate endothelial eNOS-activating pathways and NO production. As a result, the endothelial antiinflammatory and endothelial repair effects of HDL are impaired. Notably, in contrast to HDL from healthy subjects, HDL from patients with CAD is characterized by a gain of stimulatory effects on endothelial LOX-1 and PKC $\beta$ II that inhibit eNOS-activating pathways. Moreover, our findings suggest that impaired HDL-associated PON1 activity plays a role in endothelial activation of PKC $\beta I$ II by HDL from patients with CAD as well as for impaired effects of HDL from patients with CAD on eNOSactivating pathways and eNOS-dependent endothelial antiinflammatory and repair effects. These findings support the concept that the biological functions of HDL in addition to its plasma levels may have to be taken into account to assess the cardiovascular effects of HDL-raising therapies in patients with CAD.

\section{Methods}

A detailed description of the methods used in this study is provided in Supplemental Methods.

Patient population and blood sampling. Blood samples were obtained from patients with sCAD or ACS (ST elevation myocardial infarction [STEMI] or non-ST elevation myocardial infarction [NSTEMI]) and healthy subjects (without cardiovascular risk factors) between 40 and 70 years of age who were consecutively recruited into the study at the University Hospital of Zurich. The diagnosis of SCAD and an ACS was made according to guidelines of the American Heart Association $(69,70)$. In brief, patients with ACS (STEMI and NSTEMI) were recruited if they presented within 12 hours after the onset of symptoms and were in a fasting state for at least 12 hours. Patients were excluded from the study if there was evidence for accompanying infectious, inflammatory or autoimmune disorders, diabetes, advanced kidney or liver failure, neoplastic disorders, and a history of major surgery or trauma within the previous month. Age- and sex-matched healthy control subjects were consecutively enrolled by the Blood Donation Service of the University Hospital Zurich and had no cardiovascular risk factors (according to history, clinical examination, and laboratory tests) or accompanying disorders. All subjects gave written informed consent prior to inclusion in the study, and the study was approved by the local ethics committee (Kantonale Ethik-Kommission, Zurich, Switzerland).

Pon1- and eNOS-deficient mice. Pon1-deficient mice were produced by targeted disruption of exon 1 of the Pon 1 gene, as described previously (62), and backcrossed more than 10 times onto the C57BL/6J background. Blood for the isolation of HDL from Pon1-deficient and wild-type mice was collected from the retroorbital plexus after a fasting period of 16 hours and immediately centrifuged to obtain serum. eNOS-deficient mice were purchased from The Jackson Laboratory.

Isolation of HDL. HDL from subjects with $\mathrm{SCAD}$ or an $\mathrm{ACS}$, healthy controls, and wild-type and Pon1-deficient mice was isolated by sequential ultracentrifugation $(d=1.063-1.21 \mathrm{~g} / \mathrm{ml})$ according to the method of Havel et al. $(9,36,71)$ using solid potassium bromide (Merck) for density adjustment. Blood samples were processed within 1 hour after collection. $\mathrm{HDL}_{2}$ and $\mathrm{HDL}_{3}(d=1.110-1.210 \mathrm{~g} / \mathrm{ml})$ were separated as described previously $(9,36)$.

Measurement of endothelial cell NO production by ESR spectroscopy. NO production in human aortic endothelial cells (HAECs) was measured by ESR spectroscopy using the spin-probe colloid $\mathrm{Fe}(\mathrm{DETC})_{2}$. A detailed description of the method is provided in Supplemental Methods.

Measurement of endothelial cell NO production by 4,5-diaminofluorescein diacetate staining. HAECs were incubated with 4,5-diaminofluorescein diacetate (DAF-2; $1 \mu \mathrm{M}$; Cayman Chemical), and triazolofluorescein fluorescence was measured using an excitation wavelength of $485 \mathrm{~nm}$.

Measurement of endothelial cell superoxide production by ESR spectroscopy. Superoxide production in HAECs was measured by ESR spectroscopy using the spin probe 1-hydroxy-3-methoxycarbonyl-2,2,5,5-tetramethylpyrrolidine (CMH; final concentration $200 \mu \mathrm{M}$; Noxygen).

Measurement of $N A D(P) H$ oxidase activity in endothelial cells by ESR spectroscopy. $\mathrm{NAD}(\mathrm{P}) \mathrm{H}$ oxidase activity was measured in homogenates of HAECs by ESR spectroscopy using the spin probe 1-hydroxy-3-carboxypyrrolidine (CP-H; Noxygen).

Endothelial monocyte adhesion. CD14-positive monocytes were isolated by Ficoll density gradient centrifugation (Vacutainer CPT, BD) and labeled with CFSE (Molecular Probes, Invitrogen). HAECs were preincubated with HDL $(50 \mu \mathrm{g} / \mathrm{ml})$ for 1 hour and stimulated with TNF- $\alpha(1 \mathrm{ng} / \mathrm{ml})$ for 3 hours. Afterward, cell culture medium was changed, and monocytes (50,000/well) were added to the HAEC monolayer. After 3 hours, adherent CFSE-labeled monocytes were counted on 4 randomly selected high-power fields using a fluorescence microscope (DM-IRB, Leica).

Endothelial cell transfection with eNOS-specific small interference RNA. HAECs were transfected with eNOS-specific or scrambled siRNA at a final concentration of $15 \mathrm{nM}$ using the N-TER nanoparticle siRNA transfection system (Sigma-Aldrich). siRNA sequences are provided in Supplemental Methods.

Detection of nuclear translocation of the NF- $\mathrm{\kappa} B$ subunit RelA/p65 by immunocytochemistry and quantification of DNA-bound $p 65$ in nuclear extracts of endothelial cells. Nuclear translocation of the NF-אB subunit RelA/p65 was detected by staining cells with mouse anti-RelA/p65 (Santa Cruz Biotechnology Inc.) and analyzing the amount of cytoplasmic RelA/p65 by an SP2 confocal microscope (Leica). Binding of activated NF- $\mathrm{KB}$ in the nuclear extracts to an oligonucleotide containing an NF-KB consensus binding site was detected by a RelA/p65 transcription factor assay (TransAM kit, Active Motif).

In vivo endothelial repair assay. Male $N R M I^{n u / n u}$ athymic nude mice, aged 7-10 weeks, were used to allow injection of HDL (15 mg HDL protein $/ \mathrm{kg}$ body weight). Animals were anesthetized with ketamine (100 mg/kg i.p.) and xylazine (5 $\mathrm{mg} / \mathrm{kg}$ i.p.). Carotid artery electric injury was performed as described previously $(36,42,43)$. In brief, the left common carotid artery was injured with a bipolar microregulator (ICC 50, ERBE-Elektromedizin $\mathrm{GmbH})$. An electric current of $2 \mathrm{~W}$ was applied for 2 seconds to each millimeter of carotid artery over a total length of exactly $4 \mathrm{~mm}$ with the use of a size marker parallel to the carotid artery. HDL was injected 3 hours after 
carotid injury via tail vein injection with a 27 -gauge needle. The same volume of PBS was injected into control mice. Three days after carotid injury, endothelial repair was evaluated by staining denuded areas with $50 \mu \mathrm{l}$ of solution containing $5 \%$ Evans blue dye via tail vein injection. The re-endothelialized area was calculated as the difference between the blue-stained area and the injured area by computer-assisted morphometric analysis. Of note, this model has been shown to achieve accurate quantification of endothelial repair $(36,42,43)$. All animal protocols were approved by the animal care and use committee (Kantonales Veterinäramt, Zurich, Switzerland).

Binding of HDL to endothelial cells. Binding of HDL to HAECs was examined as described in detail previously $(36,44)$. Further details are provided in Supplemental Methods.

Characterization of cellular cholesterol efflux capacity of HDL. Total and ABCA1-dependent efflux of $\left[{ }^{14} \mathrm{C}\right]$ cholesterol to HDL was measured using J774 macrophages treated with or without the cAMP analog 8-(4-chlorophenylthio)adenosine-3',5'-cyclic monophosphate (cpt-cAMP). The ABCG1dependent cholesterol efflux capacity of HDL was measured in HEK293 cells transfected with an ABCG1-expressing plasmid or a mock plasmid.

Inbibition of endothelial LOX-1 by blocking antibody. The endothelial LOX-1 receptor was inhibited by a commercially available blocking antibody (R\&D Systems), as described previously (72). In brief, HAECs were incubated with the blocking antibody $(15 \mu \mathrm{g} / \mathrm{ml})$ for 2 hours, and afterward endothelial cells were subjected to ESR spectroscopy or Western blot analysis.

Detection of protein-bound MDA in HDL by spectrophotometry. Free and protein-bound MDA in HDL was detected by a commercially available lipid peroxidation assay kit (ALDetect, Enzo Life Sciences) involving an acid hydrolysis step in the presence of butylated hydroxytoluene (BHT) to allow the quantification of Schiff base MDA adducts. Hydrolysis of Schiff base MDA adducts in HDL was carried out after addition of hydrochloric acid at $\mathrm{pH} 1-2$ and heating at $60^{\circ} \mathrm{C}$ for 80 minutes.

Characterization of MDA-lysine adducts in HDL. HDL was digested with trypsin or endoproteinase Glu-C and fractionated by liquid chromatography. MDA-lysine adducts were analyzed by MS/MS analysis, as described in detail in Supplemental Methods.

MDA modification of HDL. MDA was freshly prepared by rapid acid hydrolysis of malonaldehyde bis-(dimethyl acetal) and used for in vitro modification of HDL according to previously published protocols (33). Briefly, maloncarbonyl bis-(dimethylacetal) was mixed at $1: 10$ with $1 \mathrm{M} \mathrm{HCl}$ and incubated for 1 hour at $50^{\circ} \mathrm{C}$ under gentle agitation. The reaction mixture was dissolved with phosphate buffer (10 mM, pH 7.4), and the concentration of MDA was measured spectrophotometrically, as described previously (33). HDL ( $0.2 \mathrm{mg}$ protein $/ \mathrm{ml}$ ) was incubated with freshly prepared MDA for 24 hours at $37^{\circ} \mathrm{C}$ in $50 \mathrm{mM}$ sodium phosphate buffer ( $\mathrm{pH} 7.4$ ) containing $100 \mu \mathrm{m}$ DTPA.

Measurement of PON1 activity and content of HDL. Paraoxonase and arylesterase activities of HDL-associated PON1 were measured by spectrophotometry using the substrates paraoxon and phenylacetate, respectively. The content of PON1 in HDL was measured by Western blot analysis.

Inbibition of PON1 and supplementation of HDL with purified human PON1. HDL-associated PON1 was inhibited by hydroxyquinoline or EDTA, as described previously $(53,54)$. HDL was supplemented with human purified PON1 at a dose of $5 \mathrm{U} / 100 \mu \mathrm{g}$ HDL protein.

Statistics. All data are expressed as mean \pm SEM. Statistical comparisons were made by the nonparametric Wilcoxon 2 -sample test (paired analysis) or the nonparametric Mann-Whitney $U$ test, and a $P$ value less than 0.05 was considered statistically significant. Bonferroni adjustment was performed in the comparisons of the clinical cohorts. All analyses were performed with GraphPad Prism software (version 4.0).

\section{Acknowledgments}

We thank Bernd Roschitzki (Functional Genomics Center Zurich), Ines Bühler, Simone Kaufmann, and the staff of the Andreas Grüntzig Catheterization Laboratory at the University Hospital Zurich for their excellent technical assistance. This work was supported by two Swiss National Research Foundation grants (310030-122339 and 3100A0-116404/1), the Swiss Heart Foundation, the Zurich Center for Integrative Human Physiology, the Roche Research Foundation, an educational grant from Pfizer, the Leducq Foundation, the European Union (HDLomics: LSHM-CT2006-037631), and U.S. Public Health Service grant HL-30568.

Received for publication December 13, 2010, and accepted in revised form May 4, 2011.

Address correspondence to: Ulf Landmesser, Cardiology, Cardiovascular Center, University Hospital Zurich, Raemistrasse 100, 8091 Zurich, Switzerland. Phone: 0041.44.255.9595; Fax: 0041.44.255.4251; E-mail: Ulf.Landmesser@usz.ch.
1. Di Angelantonio E, et al. Major lipids, apolipoproteins, and risk of vascular disease. JAMA. 2009; 302(18):1993-2000.

2. Barter P, et al. HDL cholesterol, very low levels of LDL cholesterol, and cardiovascular events. $N$ Engl J Med. 2007;357(13):1301-1310.

3. Rader DJ. Molecular regulation of HDL metabolism and function: implications for novel therapies. J Clin Invest. 2006;116(12):3090-3100.

4. Tall AR, Yvan-Charvet L, Terasaka N, Pagler T, Wang N. HDL, ABC transporters, and cholesterol efflux: implications for the treatment of atherosclerosis. Cell Metab. 2008;7(5):365-375.

5. Mineo C, Deguchi H, Griffin JH, Shaul PW. Endothelial and antithrombotic actions of HDL. Circ Res. 2006;98(11):1352-1364.

6. von Eckardstein A, Rohrer L. Transendothelial lipoprotein transport and regulation of endothelial permeability and integrity by lipoproteins. Curr Opin Lipidol. 2009;20(3):197-205.

7. Yuhanna IS, et al. High-density lipoprotein binding to scavenger receptor-BI activates endothelial nitric oxide synthase. Nat Med. 2001;7(7):853-857.

8. Mineo C, Yuhanna IS, Quon MJ, Shaul PW. High density lipoprotein-induced endothelial nitricoxide synthase activation is mediated by Akt and
MAP kinases. J Biol Chem. 2003;278(11):9142-9149.

9. Nofer JR, et al. HDL induces NO-dependent vasorelaxation via the lysophospholipid receptor S1P3. J Clin Invest. 2004;113(4):569-581.

10. Terasaka N, et al. ABCG1 and HDL protect against endothelial dysfunction in mice fed a high-cholesterol diet. J Clin Invest. 2008;118(11):3701-3713.

11. Seetharam D, et al. High-density lipoprotein promotes endothelial cell migration and reendothelialization via scavenger receptor-B type I. Circ Res. 2006;98(1):63-72.

12. Tso C, Martinic G, Fan WH, Rogers C, Rye KA, Barter PJ. High-density lipoproteins enhance progenitor-mediated endothelium repair in mice. Arterioscler Thromb Vasc Biol. 2006;26(5):1144-1149.

13. Noor R, et al. High-density lipoprotein cholesterol regulates endothelial progenitor cells by increasing eNOS and preventing apoptosis. Atherosclerosis. 2007;192(1):92-99.

14. Feng Y, et al. Human ApoA-I transfer attenuates transplant arteriosclerosis via enhanced incorporation of bone marrow-derived endothelial progenitor cells. Arterioscler Thromb Vasc Biol. 2008;28(2):278-283.

15. Petoumenos V, Nickenig G, Werner N. High density lipoprotein exerts vasculoprotection via endothelial progenitor cells. J Cell Mol Med. 2009;
13(11-12):4623-4635.

16. Assanasen C, et al. Cholesterol binding, efflux, and a PDZ-interacting domain of scavenger receptor-BI mediate HDL-initiated signaling. J Clin Invest. 2005; 115(4):969-977.

17. Saddar S, Mineo C, Shaul PW. Signaling by the highaffinity HDL receptor scavenger receptor B type I. Arterioscler Thromb Vasc Biol. 2010;30(2):144-150.

18. Terasaka N, et al. ATP-binding cassette transporter G1 and high-density lipoprotein promote endothelial NO synthesis through a decrease in the interaction of caveolin-1 and endothelial NO synthase. Arterioscler Thromb Vasc Biol. 2010;30(11):2219-2225.

19. Spieker LE, et al. High-density lipoprotein restores endothelial function in hypercholesterolemic men. Circulation. 2002;105(12):1399-1402.

20. Bisoendial RJ, et al. Restoration of endothelial function by increasing high-density lipoprotein in subjects with isolated low high-density lipoprotein. Circulation. 2003;107(23):2944-2948.

21. Shaul PW, Mineo C. HDL action on the vascular wall: is the answer NO? J Clin Invest. 2004;113(4):509-513.

22. Lusis AJ. Atherosclerosis. Nature. 2000; 407(6801):233-241.

23. Landmesser U, Hornig B, Drexler H. Endothelial function: a critical determinant in atherosclerosis? 
Circulation. 2004;109(21 suppl 1):II27-33.

24. Forstermann U, Munzel T. Endothelial nitric oxide synthase in vascular disease: from marvel to menace. Circulation. 2006;113(13):1708-1714.

25 . De Caterina R, et al. Nitric oxide decreases cytokineinduced endothelial activation. Nitric oxide selectively reduces endothelial expression of adhesion molecules and proinflammatory cytokines. J Clin Invest. 1995;96(1):60-68.

26. Kuhlencordt PJ, et al. Accelerated atherosclerosis, aortic aneurysm formation, and ischemic heart disease in apolipoprotein $\mathrm{E} / \mathrm{endothelial} \mathrm{nitric} \mathrm{oxide}$ synthase double-knockout mice. Circulation. 2001; 104(4):448-454.

27. Qian H, Neplioueva V, Shetty GA, Channon KM George SE. Nitric oxide synthase gene therapy rapidly reduces adhesion molecule expression and inflammatory cell infiltration in carotid arteries of cholesterolfed rabbits. Circulation. 1999;99(23):2979-2982.

28. Rader DJ. Illuminating HDL - is it still a viable therapeutic target? NEnglJ Med. 2007;357(21):2180-2183.

29. Tall AR. CETP inhibitors to increase HDL cholesterol levels. NEngl J Med. 2007;356(13):1364-1366.

30. Duffy D, Rader DJ. Update on strategies to increase HDL quantity and function. Nat Rev Cardiol. 2009; 6(7):455-463.

31. Barter PJ, et al. Effects of torcetrapib in patients at high risk for coronary events. $N$ Engl J Med. 2007; 357(21):2109-2122.

32. Zheng L, et al. Apolipoprotein A-I is a selective target for myeloperoxidase-catalyzed oxidation and functional impairment in subjects with cardiovascular disease. J Clin Invest. 2004;114(4):529-541.

33. Shao B, et al. Modifying apolipoprotein A-I by malondialdehyde, but not by an array of other reactive carbonyls, blocks cholesterol efflux by the ABCA1 pathway. J Biol Chem. 2010;285(24):18473-18484.

34. Navab M, et al. Monocyte transmigration induced by modification of low density lipoprotein in cocultures of human aortic wall cells is due to induction of monocyte chemotactic protein 1 synthesis and is abolished by high density lipoprotein. J Clin Invest. 1991;88(6):2039-2046.

35. Ansell BJ, et al. Inflammatory/antiinflammatory properties of high-density lipoprotein distinguish patients from control subjects better than high-density lipoprotein cholesterol levels and are favorably affected by simvastatin treatment. Circulation. 2003; 108(22):2751-2756.

36. Sorrentino SA, et al. Endothelial-vasoprotective effects of high-density lipoprotein are impaired in patients with type 2 diabetes mellitus but are improved after extended-release niacin therapy. Circulation. 2010;121(1):110-122.

37. Charakida $M$, et al. Vascular abnormalities, paraoxonase activity, and dysfunctional HDL in primary antiphospholipid syndrome. JAMA. 2009; 302(11):1210-1217.

38. Fleming I, Fisslthaler B, Dimmeler S, Kemp BE, Busse R. Phosphorylation of $\operatorname{Thr}(495)$ regulates $\mathrm{Ca}(2+) /$ calmodulin-dependent endothelial nitric oxide synthase activity. Circ Res. 2001;88(11):E68-E75.

39. Cai H, Harrison DG. Endothelial dysfunction in cardiovascular diseases: the role of oxidant stress. Circ Res. 2000;87(10):840-844

40. Dimmeler S, Fleming I, Fisslthaler B, Hermann C, Busse R, Zeiher AM. Activation of nitric oxide synthase in endothelial cells by Akt-dependent phosphorylation. Nature. 1999;399(6736):601-605.

41. Collins T, Cybulsky MI. NF-kappaB: pivotal mediator or innocent bystander in atherogenesis? J Clin Invest. 2001;107(3):255-264.

42. Brouchet L, Krust A, Dupont S, Chambon P,
Bayard F, Arnal JF. Estradiol accelerates reendothelialization in mouse carotid artery through estrogen receptor-alpha but not estrogen receptor-beta. Circulation. 2001;103(3):423-428.

43. Sorrentino SA, et al. Oxidant stress impairs in vivo reendothelialization capacity of endothelial progenitor cells from patients with type 2 diabetes mellitus: restoration by the peroxisome proliferator-activated receptor-gamma agonist rosiglitazone. Circulation. 2007;116(2):163-173.

44. Rohrer L, Ohnsorg PM, Lehner M, Landolt F, Rinninger F, von Eckardstein A. High-density lipoprotein transport through aortic endothelial cells involves scavenger receptor $\mathrm{BI}$ and ATP-binding cassette transporter G1. Circ Res. 2009;104(10):1142-1150.

45. Beckman JA, Goldfine AB, Gordon MB, Garrett LA, Creager MA. Inhibition of protein kinase Cbeta prevents impaired endothelium-dependent vasodilation caused by hyperglycemia in humans. Circ Res. 2002;90(1):107-111.

46. Kuboki K, et al. Regulation of endothelial constitutive nitric oxide synthase gene expression in endothelial cells and in vivo: a specific vascular action of insulin. Circulation. 2000;101(6):676-681.

47. Naruse K, et al. Activation of vascular protein kinase C-beta inhibits Akt-dependent endothelial nitric oxide synthase function in obesity-associated insulin resistance. Diabetes. 2006;55(3):691-698.

48. Payne GA, Bohlen HG, Dincer UD, Borbouse L, Tune JD. Periadventitial adipose tissue impairs coronary endothelial function via PKC-beta-dependent phosphorylation of nitric oxide synthase. Am J Physiol Heart Circ Physiol. 2009;297(1):H460-H465.

49. Kouroedov A, Eto M, Joch H, Volpe M, Luscher TF, Cosentino F. Selective inhibition of protein kinase Cbeta2 prevents acute effects of high glucose on vascular cell adhesion molecule-1 expression in human endothelial cells. Circulation. 2004;110(1):91-96.

50. Li D, Liu L, Chen H, Sawamura T, Ranganathan S, Mehta JL. LOX-1 mediates oxidized low-density lipoprotein-induced expression of matrix metalloproteinases in human coronary artery endothelial cells. Circulation. 2003;107(4):612-617.

51. Marsche G, Levak-Frank S, Quehenberger O, Heller R, Sattler W, Malle E. Identification of the human analog of SR-BI and LOX-1 as receptors for hypochlorite-modified high density lipoprotein on human umbilical venous endothelial cells. FASEBJ. 2001;15(6):1095-1097.

52. Aviram M, Rosenblat M, Bisgaier CL, Newton RS, Primo-Parmo SL, La Du BN. Paraoxonase inhibits high-density lipoprotein oxidation and preserves its functions. A possible peroxidative role for paraoxonase. J Clin Invest. 1998;101(8):1581-1590.

53. Aviram M, Rosenblat M. Paraoxonases (PON1, PON2, PON3) analyses in vitro and in vivo in relation to cardiovascular diseases. Methods Mol Biol. 2008; 477:259-276.

54. Rosenblat M, Vaya J, Shih D, Aviram M. Paraoxonase 1 (PON1) enhances HDL-mediated macrophage cholesterol efflux via the ABCA1 transporter in association with increased HDL binding to the cells: a possible role for lysophosphatidylcholine. Atherosclerosis. 2005;179(1):69-77.

55. Barter PJ, Nicholls S, Rye KA, Anantharamaiah GM, Navab M, Fogelman AM. Antiinflammatory properties of HDL. Circ Res. 2004;95(8):764-772.

56. Cybulsky MI, et al. A major role for VCAM-1, but not ICAM-1, in early atherosclerosis. J Clin Invest. 2001;107(10):1255-1262.

57. Rader DJ, Daugherty A. Translating molecular discoveries into new therapies for atherosclerosis. Nature. 2008;451(7181):904-913.
58. Kawakami A, et al. Apolipoprotein CIII links hyperlipidemia with vascular endothelial cell dysfunction. Circulation. 2008;118(7):731-742.

59. Aviram $M$, et al. Human serum paraoxonases (PON1) Q and R selectively decrease lipid peroxides in human coronary and carotid atherosclerotic lesions: PON1 esterase and peroxidase-like activities. Circulation. 2000;101(21):2510-2517.

60. Watson AD, et al. Protective effect of high density lipoprotein associated paraoxonase. Inhibition of the biological activity of minimally oxidized low density lipoprotein. JClin Invest. 1995;96(6):2882-2891.

61. Undurti A, Huang Y, Lupica JA, Smith JD, DiDonato JA, Hazen SL. Modification of high density lipoprotein by myeloperoxidase generates a pro-inflammatory particle. J Biol Chem. 2009;284(45):30825-30835.

62. Shih DM, et al. Mice lacking serum paraoxonase are susceptible to organophosphate toxicity and atherosclerosis. Nature. 1998;394(6690):284-287.

63. Tward A, et al. Decreased atherosclerotic lesion formation in human serum paraoxonase transgenic mice. Circulation. 2002;106(4):484-490.

64. Bhattacharyya T, et al. Relationship of paraoxonase 1 (PON1) gene polymorphisms and functional activity with systemic oxidative stress and cardiovascular risk. JAMA. 2008;299(11):1265-1276.

65. Regieli JJ, et al. Paraoxonase variants relate to 10 -year risk in coronary artery disease: impact of a high-density lipoprotein-bound antioxidant in secondary prevention. J Am Coll Cardiol. 2009;54(14):1238-1245.

66 . Bergt $\mathrm{C}$, et al. The myeloperoxidase product hypochlorous acid oxidizes HDL in the human artery wall and impairs ABCA1-dependent cholesterol transport. Proc Natl Acad Sci US A. 2004;101(35):13032-13037.

67. Lerman A, Zeiher AM. Endothelial function: cardiac events. Circulation. 2005;111(3):363-368.

68. Watanabe J, et al. Hemoglobin and its scavenger protein haptoglobin associate with apoA-1-containing particles and influence the inflammatory properties and function of high density lipoprotein. J Biol Chem. 2009;284(27):18292-18301.

69. Anderson JL, et al. ACC/AHA 2007 guidelines for the management of patients with unstable angina/non ST-elevation myocardial infarction: a report of the American College of Cardiology/American Heart Association Task Force on Practice Guidelines (Writing Committee to Revise the 2002 Guidelines for the Management of Patients With Unstable Angina/ Non ST-Elevation Myocardial Infarction): developed in collaboration with the American College of Emergency Physicians, the Society for Cardiovascular Angiography and Interventions, and the Society of Thoracic Surgeons: endorsed by the American Association of Cardiovascular and Pulmonary Rehabilitation and the Society for Academic Emergency Medicine. Circulation. 2007;116(7):e148-e304.

70. Antman EM, et al. ACC/AHA guidelines for the management of patients with ST-elevation myocardial infarction: a report of the American College of Cardiology/American Heart Association Task Force on Practice Guidelines (Committee to Revise the 1999 Guidelines for the Management of Patients with Acute Myocardial Infarction). Circulation. 2004;110(9):e82-e292.

71. Havel RJ, Eder HA, Bragdon JH. The distribution and chemical composition of ultracentrifugally separated lipoproteins in human serum.J Clin Invest. 1955;34(9):1345-1353.

72. Thum T, Borlak J. LOX-1 receptor blockade abrogates oxLDL-induced oxidative DNA damage and prevents activation of the transcriptional repressor Oct-1 in human coronary arterial endothelium. J Biol Chem. 2008;283(28):19456-19464. 